\title{
RUIN PROBABILITY WITH CERTAIN STATIONARY STABLE CLAIMS GENERATED BY CONSERVATIVE FLOWS
}

\author{
UĞUR TUNCAY ALPARSLAN, ${ }^{*}$ University of Nevada, Reno \\ GENNADY SAMORODNITSKY, ${ }^{* *}$ Cornell University
}

\begin{abstract}
We study the ruin probability where the claim sizes are modeled by a stationary ergodic symmetric $\alpha$-stable process. We exploit the flow representation of such processes, and we consider the processes generated by conservative flows. We focus on two classes of conservative $\alpha$-stable processes (one discrete-time and one continuous-time), and give results for the order of magnitude of the ruin probability as the initial capital goes to infinity. We also prove a solidarity property for null-recurrent Markov chains as an auxiliary result, which might be of independent interest.
\end{abstract}

Keywords: Ruin probability; stable process; heavy tail; ergodic theory; long range dependence; conservative flow; null-recurrent Markov chain; fractional Brownian motion

2000 Mathematics Subject Classification: Primary 60G52

Secondary 62P05

\section{Introduction}

One of the popular problems of applied probability involves analyzing the exceedance probability of a threshold $u$ given by

$$
\psi(u)=\mathrm{P}\left(\sup _{t \in \mathbb{T}}(S(t)-\mu(t))>u\right),
$$

where $S=\{S(t), t \in \mathbb{T}\}$ is a random walk and $\mu=\{\mu(t), t \in \mathbb{T}\}$ is a nonrandom drift term with an index set $\mathbb{T}$. This quantity has various interpretations in several different fields. In the context of risk theory and insurance, $\boldsymbol{S}$ can be viewed as the cumulative claim size process, whereas $\mu$ can be viewed as cumulative premium income on the insurance policy. In this case, we can view the exceedance probability as the ruin probability with initial capital $u$, or as the ruin probability, for short. (See [10, pp. 22-24].)

In this study we adhere to the language of insurance, however casually, although the results can be easily interpreted in other fields, including (but not limited to) queueing and storage/dam models.

Research on ruin probabilities, in the sense of modern actuarial science, was mainly initiated in Sweden in the first half of the 20th century. The foundations of the theory were laid down by Filip Lundberg in his Uppsala thesis (see [13]), while the first mathematically substantial results

Received 4 January 2007.

* Postal address: Department of Mathematics and Statistics, University of Nevada, Reno, NV 89557, USA.

Email address: augur@unr.edu

** Postal address: School of Operations Research and Industrial Engineering, Cornell University, Ithaca, NY 14853, USA. 
appeared in a series of papers by Lundberg and Harald Cramér. (See [6], [7], [14], and [15].) The basic model coming out of these first contributions is widely referred to as the Cramér-Lundberg model (for details see, for instance, [10, p. 22]). Since then there have been numerous extensions of the classical Cramér-Lundberg model with independent, identically distributed (i.i.d.), lighttailed claim sizes. More recently however, work in this area has turned to the more realistic setting of dependent claims. Moreover, empirical evidence in fields including insurance and financial markets, and the effort by banks, insurance companies, and governmental institutions to control risk associated with extreme events resulting in 'large claims' has led to the theoretical interest in modeling 'heavy tailed' phenomena.

In addition, from a theoretical point, the case of heavy-tailed, dependent claims is also interesting as it raises the question of the possibility of relating the dependence structure of the heavy-tailed stationary processes underlying the claims to the asymptotic behavior of the ruin probability. This becomes particularly challenging when the second moment of the claim sizes is infinite, so that it is not possible to use covariances to quantify the strength and the range of dependence.

In this study we focus on claim sizes modeled by stationary ergodic symmetric $\alpha$-stable ( $\mathrm{S} \alpha \mathrm{S})$ processes, an important class of heavy-tailed processes. We choose to work with $\alpha \in(1,2)$, for which the claim process has a finite first moment but infinite second moment, and the ruin probability with a linear premium process is nontrivial. This, together with the fact that the probabilistic structure of these processes is relatively well understood, allows us to focus on the underlying dependence structure in the presence of heavy tails.

Throughout this paper we will assume a constant premium rate, (i.e. a linear drift term).

The setup of $S \alpha S$ claims with $\mathbb{T}=\mathbb{Z}_{+}$, deterministic claim arrival processes, and constant premium rates has been addressed by Mikosch and Samorodnitsky [16], which is the origin of our current work. Based on the results of [9], the authors have observed that the order of magnitude of $\psi(u)$ for this model is $u^{-(\alpha-1)}$ in the case of i.i.d. claim sizes. Therefore, this is the 'fastest' rate we can expect the ruin probability to decay in such a model. It is also shown ibid that for certain claim processes $\psi(u)$ decays as fast as $u^{-(\alpha-1)}$ even when the claim sizes are dependent. In the tradition of Mikosch and Samorodnitsky, we think of claim processes in this class as short-range dependent. They also showed that for certain classes of $\mathrm{S} \alpha \mathrm{S}$ claims, $\psi(u)$ may decay at a slower rate than $u^{-(\alpha-1)}$. We think of these processes as long-range dependent.

In this study, we also investigate the case of $\mathbb{T}=\mathbb{R}_{+}$utilizing recent results of [4].

Now let our claim process, $\boldsymbol{X}=\{X(t), t \in \mathbb{T}\}$, be a measurable stationary ergodic $\mathrm{S} \alpha \mathrm{S}$ process with $\alpha \in(1,2)$ given in the form

$$
X(t)=\int_{E} f_{t}(x) M(\mathrm{~d} x), \quad t \in \mathbb{T},
$$

where $M$ is a $\mathrm{S} \alpha \mathrm{S}$ random measure on a measurable space $(E, \mathcal{E})$ with a $\sigma$-finite control measure $m$ on $\mathcal{E}$ (i.e. $M$ is an independently scattered random measure on $\mathcal{E}$ such that

$$
E \exp \{i \lambda M(A)\}=\exp \left\{-|\lambda|^{\alpha} m(A)\right\}, \quad \lambda \in \mathbb{R},
$$

for every $A \in \mathcal{E}$, with $m(A)<\infty$, and $\left\{f_{t}\right\}_{t \in \mathbb{T}} \subset L^{\alpha}(E, \mathcal{E}, m)$. (See Section 3.3 of [23].)

As we consider stationary $\mathrm{S} \alpha \mathrm{S}$ processes we can choose $f_{t}$ to be in a particularly descriptive form given by

$$
f_{t}(x)=a_{t}(x)\left[\frac{\mathrm{d} m \circ \phi_{t}}{\mathrm{~d} m}(x)\right]^{\alpha} f \circ \phi_{t}(x), \quad x \in E, t \in \mathbb{T},
$$


where $\left\{\phi_{t}\right\}_{t \in \mathbb{T}}$ is a nonsingular flow (recall that a flow is a family of measurable maps from $E$ onto $E$ such that $\phi_{t_{1}+t_{2}}=\phi_{t_{1}} \circ \phi_{t_{2}}$ for all $t_{1}, t_{2} \in \mathbb{T}$, and $\phi_{0}$ is the identity function on $E$ ), $\left\{a_{t}\right\}_{t \in \mathbb{T}}$ is a cocycle for this flow (i.e. for every $t_{1}, t_{2} \in \mathbb{T}, a_{t_{1}+t_{2}}(x)=a_{t_{2}}(x) a_{t_{1}} \circ \phi_{t_{2}}(x)$ for $m$-almost all $x \in E)$ taking values in $\{-1,1\}$, and $f \in L^{\alpha}(E, \mathcal{E}, m)$. (See [20].)

This representation is particularly important as it brings up the possibility of relating the properties of a stationary $\mathrm{S} \alpha \mathrm{S}$ process to those of a flow and a single kernel. For instance, Hopf decomposition (see, e.g. [12, pp. 17, 116]) of the flow $\left\{\phi_{t}\right\}_{t \in T}$ immediately implies that a stationary $\mathrm{S} \alpha \mathrm{S}$ process, $\boldsymbol{X}$, can be written (in distribution) as a sum of two independent stationary $\mathrm{S} \alpha \mathrm{S}$ processes

$$
\boldsymbol{X}=\boldsymbol{X}^{\mathrm{D}}+\boldsymbol{X}^{\mathrm{C}}
$$

where $\boldsymbol{X}^{\mathrm{D}}$ is given by representations (1.2) and (1.3) with a dissipative flow, and $\boldsymbol{X}^{\mathrm{C}}$ is given by representations (1.2) and (1.3) with a conservative flow.

In this paper we investigate the asymptotic behavior of the ruin probability when the claims constitute a stationary $\mathrm{S} \alpha \mathrm{S}$ process generated purely by conservative flows, i.e. processes of the form $\boldsymbol{X}^{\mathrm{C}}$ given in (1.4).

The case of stationary $\mathrm{S} \alpha \mathrm{S}$ claims of the form $\boldsymbol{X}^{\mathrm{D}}$ is analyzed in a separate study and the results are presented in [2].

Intuitively, we expect the range of dependence of a stationary $\mathrm{S} \alpha \mathrm{S}$ process generated by a conservative flow to be longer than that of a stationary $\mathrm{S} \alpha \mathrm{S}$ process generated by a dissipative flow. Although a complete theory of risk processes with claims associated with conservative flows is lacking at the time of this study, and in general construction of processes generated by conservative flows is not effortless, factual support for such an intuition is provided by an example investigated in [16]. In their paper the authors observed a class of conservative $\mathrm{S} \alpha \mathrm{S}$ processes constructed through a null-recurrent Markov chain (see [21] for details), and examined the asymptotic behavior of the ruin probability in a setting where the claims are modeled as a special case of this class and the premium process is a deterministic linear drift. Their results showed that the ruin probability $\psi(u)$ in this case may decay at a much slower rate than $u^{-(\alpha-1)}$ even when the kernel in the integral representation (1.2) is 'nice', i.e. in the context of ruin probabilities, at least the class of processes associated with conservative flows investigated in their example may be long-range dependent regardless of the kernel. This is indeed a significant observation as the results given in [2] suggest that in the risk theory context, for claims generated by dissipative flows, the kernel in the integral representation of the claim process is the key factor in determining the range of dependence for the process.

In Section 2 of this paper we focus on a related, but more general class of $\mathrm{S} \alpha \mathrm{S}$ processes constructed in [21], and studied in [16]. Our main result, which shows that the order of magnitude of the ruin probability $\psi(u)$ in the setting we describe below is $u^{-\gamma(\alpha-1)} L(u)$, where $L(\cdot)$ is a slowly varying function and $\gamma \in(0,1)$, is a generalization of the result given in [16]. We also prove a solidarity property for null-recurrent Markov chains as a subsidiary result, which might be of independent interest.

In Section 3, we study the ruin probability in continuous time. In particular, we concentrate on a class of stationary $\mathrm{S} \alpha \mathrm{S}$ processes associated with conservative flows constructed using a fractional Brownian motion in [22]. We use a Brownian motion to construct our claim process and we show that in this setting the order of magnitude of the ruin probability $\psi(u)$ is given by $u^{-(\alpha-1) / 2}$. We also conjecture that for a claim process associated with a fractional Brownian motion with self-similarity exponent $H \in(0,1)$, the order of magnitude is $u^{-H(\alpha-1)}$. 


\section{A discrete time claim process associated with a conservative flow}

\subsection{Setup and assumptions}

Consider an irreducible, null-recurrent Markov chain, $\boldsymbol{Y}=\left\{Y_{n}, n \geq 1\right\}$, on $\mathbb{Z}$ with law $\mathrm{P}_{S}(\cdot)$ on

$$
E=\left\{\boldsymbol{y}=\left(y_{0}, y_{1}, y_{2}, \ldots\right): y_{i} \in \mathbb{Z}, i=0,1,2, \ldots\right\}
$$

corresponding to the initial state $y_{0}=s \in \mathbb{Z}$.

Let $\pi=\left\{\pi_{s}, s \in \mathbb{Z}\right\}$ be the $\sigma$-finite invariant measure corresponding to the family $\left\{\mathrm{P}_{s}, s \in \mathbb{Z}\right\}$ satisfying $\pi_{0}=1$, and define a $\sigma$-finite measure on the cylindrical $\sigma$-field of $E$ by

$$
m(\cdot)=\sum_{i=-\infty}^{\infty} \pi_{i} \mathrm{P}_{i}(\cdot)
$$

Note that this measure is invariant under the shift operator $\theta: E \rightarrow E$, i.e.

$$
\theta(\boldsymbol{y})=\left(y_{1}, y_{2}, \ldots\right), \quad \boldsymbol{y}=\left(y_{0}, y_{1}, y_{2}, \ldots\right) \in E .
$$

We will model the claim size process, $X=\left\{X_{n}, n \geq 1\right\}$, with a $\mathrm{S} \alpha \mathrm{S}$ process defined by

$$
X_{n}=\int_{E} f_{n}(\boldsymbol{y}) M(\mathrm{~d} \boldsymbol{y}), \quad \boldsymbol{y} \in E, n=1,2,3, \ldots,
$$

where $M$ is a $\mathrm{S} \alpha \mathrm{S}$ random measure on $E$ with control measure $m$ given in (2.1), kernels $f_{n}$ are given by

$$
f_{n}(\boldsymbol{y})=\sum_{s \in A} a_{s} \mathbf{1}_{\left\{y_{n}=s\right\}}, \quad n \geq 1, \boldsymbol{y}=\left(y_{0}, y_{1}, y_{2}, \ldots\right) \in E,
$$

$A \subset \mathbb{Z}$ is a finite set, and $\left\{a_{s}, s \in A\right\}$ are positive real numbers. To avoid triviality assume that $A \neq \varnothing$.

It follows from [21] that the process $\boldsymbol{X}$ given by the stochastic integral representation (2.2) is a stationary mixing process, and in particular is ergodic; furthermore, $\boldsymbol{X}$ is associated with a conservative flow.

For a given $\boldsymbol{y} \in E$ and $s \in \mathbb{Z}$, define the number of steps until the chain returns to state $s$ for the first time as

$$
\tau_{s}=\tau_{s}(\boldsymbol{y}):=\inf \left\{n \geq 1: y_{n}=s\right\} .
$$

Note that by the null-recurrence of the Markov chain $E_{s} \tau_{s}=\infty$, for any $s \in \mathbb{Z}$. We will further assume that there is a constant $\gamma \in(0,1)$ and a slowly varying function $L$ such that

$$
\mathrm{P}_{0}\left(\tau_{0} \geq n\right)=n^{\gamma-1} L(n) .
$$

For an integer $s$ and a given $\boldsymbol{y} \in E$, define the number of visits to state $s$ in $n$ steps to be

$$
N_{n}^{(s)}=N_{n}^{(s)}(\boldsymbol{y}):=\sum_{j=1}^{n} \mathbf{1}_{\left\{y_{j}=s\right\}}(\boldsymbol{y})
$$

and define

$$
\eta_{n}^{(s)}:=N_{n}^{(s)} n^{\gamma-1} L(n), \quad s \in \mathbb{Z}
$$


Also, for $\boldsymbol{y} \in E, s_{0}, s_{1} \in \mathbb{Z}$, and $m \geq 1$, define the time spent in state $s_{1}$ between the $(m-1)$ th and $m$ th visits to state $s_{0}$ as

$$
W_{m}^{\left(s_{0}, s_{1}\right)}=W_{m}^{\left(s_{0}, s_{1}\right)}(\boldsymbol{y}):= \begin{cases}\sum_{j=\tau_{s_{0}}^{(m-1)}}^{\tau_{s_{0}}^{(m)}-1} \mathbf{1}_{\left\{y_{j}=s_{1}\right\}}, & \tau_{s_{0}}^{(m-1)}<\infty, \\ 0, & \tau_{s_{0}}^{(m-1)}=\infty\end{cases}
$$

(here, for $s \in \mathbb{Z}, \tau_{s}^{(m)}$ is the time of $m$ th visit to state $s$ with $\tau_{s}^{(0)}=0$ ). Note that as we are considering a recurrent Markov chain, for any $m \geq 1$,

$$
\mathrm{P}_{s_{0}}\left(\tau_{s_{0}}^{(m-1)}=\infty\right)=0,
$$

and, under $\mathrm{P}_{s_{0}},\left\{W_{m}^{\left(s_{0}, s_{1}\right)}, m \geq 1\right\}$ are i.i.d. Furthermore, note that

$$
E_{s_{0}} W_{m}^{\left(s_{0}, s_{1}\right)}=\frac{\pi_{s_{1}}}{\pi_{s_{0}}}, \quad m \geq 1
$$

(see, for instance, [17, Proposition 2.12.2]).

Finally, for a constant premium rate $\mu>0$, let the cumulative premium process be given by

$$
\underline{\mu}=\left\{\mu_{n}=n \mu, n \geq 1\right\}
$$

and define the accumulated claim process $S=\left\{S_{n}, n \geq 1\right\}$ by

$$
S_{0}=0, \quad S_{n}=\sum_{i=1}^{n} X_{n}, \quad n=1,2,3, \ldots
$$

Then the ruin probability given in (1.1) can be written as

$$
\psi(u)=\mathrm{P}\left(\sup _{n \geq 0}\left(S_{n}-n \mu\right)>u\right), \quad u>0 .
$$

\subsection{A solidarity theorem for null-recurrent Markov chains and the asymptotic analysis of the ruin probability}

We start by giving a solidarity theorem regarding the tails of the return times to a state for a Markov chain with property (2.3). This result will be utilized throughout the remainder of this section, and it will be particularly important in determining the asymptotic behavior of the moments of the number of visits to a state given the initial state. Related solidarity theorems regarding the first moment of the number of visits to a state given the initial state has been given in [24]. However, Teugels's results on the first moments give the order of magnitude without calculating the exact multiplicative constant in the asymptotic form. Furthermore, his results regarding the transition probabilities assume that the slowly varying function given in (2.3) is monotone increasing. In this study we do not require this. Additionally, in our result below, we establish the exact asymptotic equivalence by specifying the multiplicative constant.

Theorem 2.1. If (2.3) holds then, for any $s \in \mathbb{Z}$,

$$
\pi_{s} \mathrm{P}_{s}\left(\tau_{s} \geq n\right) \sim \mathrm{P}_{0}\left(\tau_{0} \geq n\right) \text { as } n \rightarrow \infty
$$


Proof. For $s=0$ the result holds trivially as $\pi_{0}=1$. Now fix $s \in \mathbb{Z} \backslash\{0\}$, and for any state $\tilde{s} \in \mathbb{Z}$ let

$$
L_{n, \tilde{s}}:=\tau_{\tilde{s}}^{\left(N_{n}^{(\tilde{s})}\right)} \quad \text { and } \quad R_{n, \tilde{s}}:=\tau_{\tilde{s}}^{\left(N_{n}^{(\tilde{s})}+1\right)}
$$

be the time of the last visit to state $\tilde{s}$ before (or at) time $n$, and the time of the first visit to state $\tilde{s}$ after $n$, respectively.

Note that

$$
\sum_{m=1}^{N_{n}^{(0)}} W_{m}^{(0, s)}=N_{L_{n, 0}}^{(s)} \leq N_{n}^{(s)} \leq N_{R_{n, 0}}^{(s)}=\sum_{m=1}^{N_{n}^{(0)}+1} W_{m}^{(0, s)}
$$

In particular,

$$
\begin{aligned}
\eta_{n}^{(0)}\left[\frac{1}{N_{n}^{(0)}} \sum_{m=1}^{N_{n}^{(0)}} W_{m}^{(0, s)}\right] & =n^{\gamma-1} L(n) N_{L_{n, 0}}^{(s)} \\
& \leq \eta_{n}^{(s)} \\
& \leq n^{\gamma-1} L(n) N_{R_{n, 0}}^{(s)} \\
& =\eta_{n}^{(0)}\left[\frac{1}{N_{n}^{(0)}} \sum_{m=1}^{N_{n}^{(0)}+1} W_{m}^{(0, s)}\right]
\end{aligned}
$$

Next observe that, for any two states $s_{0}, s_{1} \in \mathbb{Z}$, it follows from Kolmogorov's strong law of large numbers that $\mathrm{P}_{s_{0}}$-almost surely (a.s.)

$$
\lim _{n \rightarrow \infty} \frac{1}{N_{n}^{\left(s_{0}\right)}} \sum_{m=1}^{N_{n}^{\left(s_{0}\right)}} W_{m}^{\left(s_{0}, s_{1}\right)}=\lim _{n \rightarrow \infty} \frac{1}{N_{n}^{\left(s_{0}\right)}} \sum_{m=1}^{N_{n}^{\left(s_{0}\right)}+1} W_{m}^{\left(s_{0}, s_{1}\right)}=E_{s_{0}} W_{1}^{\left(s_{0}, s_{1}\right)}=\frac{\pi_{s_{1}}}{\pi_{s_{0}}} .
$$

Let $\left(Z_{1-\gamma}\right)$ be a $(1-\gamma)$-stable subordinator, i.e. a positive increasing strictly $(1-\gamma)$-stable Lévy motion with

$$
E \exp \left\{i \lambda Z_{1-\gamma}(1)\right\}=\exp \left\{-C_{1-\gamma}^{-1}|\lambda|^{1-\gamma}\left(1-\mathrm{i} \tan \frac{\pi(1-\gamma)}{2}\right)\right\}, \quad \lambda \in \mathbb{R},
$$

and $C_{1-\gamma}$ is the usual constant associated with $\alpha$-stable variables with $\alpha=1-\gamma$. In other words, $Z_{1-\gamma}(1) \sim S_{1-\gamma}\left(\sigma_{0}, 1,0\right)$, where $\sigma_{0}^{1-\gamma}=\Gamma(\gamma) \cos (\pi(1-\gamma) / 2)$. In [16] it was shown that under $\mathrm{P}_{0}$

$$
\eta_{n}^{(0)} \Rightarrow Z_{1-\gamma}^{\gamma-1}(1)
$$

Thus, it follows from (2.4), (2.5), and Slutsky's theorem that

$$
\frac{1}{\pi_{s}} \eta_{n}^{(s)} \Rightarrow Z_{1-\gamma}^{\gamma-1}(1)
$$

under $\mathrm{P}_{0}$.

We next show that (2.6) holds under $\mathrm{P}_{s}$ as well. Fix $x>0$. Note that for sufficiently large $n$

$$
\mathrm{P}_{0}\left(\eta_{n}^{(s)}>x, \tau_{s} \geq n\right) \leq \mathrm{P}_{0}\left(n^{\gamma-1} L(n)>x\right)=0,
$$


and, hence, it follows from the strong Markov property that, for large $n$,

$$
\begin{aligned}
\mathrm{P}_{0}\left(\eta_{n}^{(s)}>x\right) & =\mathrm{P}_{0}\left(\eta_{n}^{(s)}>x, \tau_{s}<n\right) \\
& \leq \sum_{i=1}^{n-1} \mathrm{P}_{0}\left(n^{\gamma-1} L(n) N_{n}^{(s)}>x \mid \tau_{s}=i\right) \mathrm{P}_{0}\left(\tau_{s}=i\right) \\
& \leq \mathrm{P}_{s}\left(\eta_{n}^{(s)}+n^{\gamma-1} L(n)>x\right) \mathrm{P}_{0}\left(\tau_{s}<n\right) \\
& \leq \mathrm{P}_{s}\left(\eta_{n}^{(s)}+n^{\gamma-1} L(n)>x\right) .
\end{aligned}
$$

Therefore, we see that

$$
\lim _{n \rightarrow \infty} \mathrm{P}_{0}\left(\eta_{n}^{(s)}>x\right) \leq \liminf _{n \rightarrow \infty} \mathrm{P}_{s}\left(\eta_{n}^{(s)}>x\right)
$$

Now let $G_{s}^{0}$ be the number of visits to state $s$ before the first visit to 0 . (Observe that $G_{s}^{0}$ has a geometric distribution under $\mathrm{P}_{s}$.) Then, for $x>0$,

$$
\begin{aligned}
\mathrm{P}_{s}\left(\eta_{n}^{(s)}>x\right)= & \mathrm{P}_{s}\left(\eta_{n}^{(s)}>x, \tau_{0} \geq n\right)+\mathrm{P}_{s}\left(\eta_{n}^{(s)}>x, \tau_{0}<n\right) \\
\leq & \mathrm{P}_{S}\left(\tau_{0} \geq n\right) \\
& +\mathrm{P}_{s}\left[n^{\gamma-1} L(n) G_{s}^{0}+n^{\gamma-1} L(n)\left(N_{n}^{(s)}-G_{s}^{0}\right)>x, \tau_{0}<n\right] .
\end{aligned}
$$

Choose $\delta \in(0,1-\gamma)$. Note that, as $n \rightarrow \infty$,

$$
n^{\delta+\gamma-1} L(n) G_{s}^{0} \stackrel{\mathrm{P}_{s}}{\rightarrow} 0 .
$$

Then, as $n$ tends to infinity,

$$
\begin{aligned}
\mathrm{P}_{s}\left[n^{\gamma-1}\right. & \left.L(n) G_{s}^{0}+n^{\gamma-1} L(n)\left(N_{n}^{(s)}-G_{s}^{0}\right)>x, \tau_{0}<n\right] \\
\leq & \mathrm{P}_{S}\left(n^{\gamma-1} L(n) G_{s}^{0}>n^{-\delta}\right) \\
& \quad+\mathrm{P}_{s}\left[n^{-\delta}+n^{\gamma-1} L(n)\left(N_{n}^{(s)}-G_{s}^{0}\right)>x, \tau_{0}<n\right] \\
= & \mathrm{P}_{s}\left[n^{-\delta}+n^{\gamma-1} L(n)\left(N_{n}^{(s)}-G_{s}^{0}\right)>x, \tau_{0}<n\right]+o(1) .
\end{aligned}
$$

However, by the strong Markov property and Slutsky's theorem we have

$$
\begin{aligned}
\mathrm{P}_{s}\left[n^{-\delta}\right. & \left.+n^{\gamma-1} L(n)\left(N_{n}^{(s)}-G_{s}^{0}\right)>x, \tau_{0}<n\right] \\
& =\sum_{i=1}^{n-1} \mathrm{P}_{s}\left[n^{-\delta}+n^{\gamma-1} L(n)\left(N_{n}^{(s)}-G_{s}^{0}\right)>x, \tau_{0}=i\right] \\
& =\sum_{i=1}^{n-1} \mathrm{P}_{0}\left(n^{-\delta}+n^{\gamma-1} L(n) N_{n-i}^{(s)}>x\right) \mathrm{P}_{s}\left(\tau_{0}=i\right) \\
& \leq \mathrm{P}_{0}\left(n^{-\delta}+n^{\gamma-1} L(n) N_{n}^{(s)}>x\right) \mathrm{P}_{s}\left(\tau_{0}<n\right) \\
& \leq \mathrm{P}_{0}\left(n^{-\delta}+\eta_{n}^{(s)}>x\right) \\
& \sim \mathrm{P}_{0}\left(\eta_{n}^{(s)}>x\right) .
\end{aligned}
$$

Combining (2.8)-(2.10) we have

$$
\mathrm{P}_{s}\left(\eta_{n}^{(s)}>x\right) \leq \mathrm{P}_{0}\left(\eta_{n}^{(s)}>x\right)+o(1) .
$$

It follows from (2.7) and (2.11) that (2.6) also holds under $\mathrm{P}_{s}$. 
Now define $\hat{a}_{n}:=\inf \left\{k: \pi_{s} k^{1-\gamma} L^{-1}(k) \geq n\right\}$. Then, for $y>0$,

$$
\begin{aligned}
& \mathrm{P}_{s}\left(\frac{\tau_{s}^{(n)}}{\hat{a}_{n}} \leq y\right) \\
& \quad=\mathrm{P}_{s}\left(N_{\left[y \hat{a}_{n}\right]}^{(s)} \geq n\right) \\
& \quad=\mathrm{P}_{s}\left[\frac{1}{\pi_{s}} \eta_{\left[y \hat{a}_{n}\right]}^{(s)} \frac{\pi_{s} \hat{a}_{n}^{1-\gamma} L^{-1}\left(\hat{a}_{n}\right)}{n} \frac{L\left(\hat{a}_{n}\right)}{L\left(y \hat{a}_{n}\right)} \frac{\left(y \hat{a}_{n}\right)^{\gamma-1} L\left(y \hat{a}_{n}\right)}{\left[y \hat{a}_{n}\right]^{\gamma-1} L\left(\left[y \hat{a}_{n}\right]\right)} \geq y^{\gamma-1}\right] .
\end{aligned}
$$

By the slow variation of $L$,

$$
\lim _{n \rightarrow \infty}\left[\frac{\pi_{s} \hat{a}_{n}^{1-\gamma} L^{-1}\left(\hat{a}_{n}\right)}{n} \frac{L\left(\hat{a}_{n}\right)}{L\left(y \hat{a}_{n}\right)} \frac{\left(y \hat{a}_{n}\right)^{\gamma-1} L\left(y \hat{a}_{n}\right)}{\left[y \hat{a}_{n}\right]^{\gamma-1} L\left(\left[y \hat{a}_{n}\right]\right)}\right]=1 .
$$

Therefore, it follows from (2.6) holding under $\mathrm{P}_{s}$, Slutsky's theorem, and the self-similarity of the stable subordinator that as $n$ goes to infinity, for $y>0$,

$$
\mathrm{P}_{s}\left(\frac{\tau_{s}^{(n)}}{\hat{a}_{n}} \leq y\right) \sim \mathrm{P}\left(Z_{1-\gamma}^{\gamma-1}(1) \geq y^{\gamma-1}\right)=\mathrm{P}\left(Z_{1-\gamma}(1) \leq y\right),
$$

i.e. $\hat{a}_{n}^{-1} \tau_{s}^{(n)} \Rightarrow Z_{1-\gamma}(1)$ under $\mathrm{P}_{s}$. Consequently (see, for instance, [23, Theorem 1.8.1, p. 50]),

$$
\mathrm{P}_{S}\left(\tau_{s}>x\right)=x^{\gamma-1} \hat{L}(x),
$$

for a slowly varying function $\hat{L}$, and moreover

$$
\mathrm{P}_{s}\left(\tau_{s}>\hat{a}_{n}\right) \sim \frac{1}{n}, \quad n \rightarrow \infty .
$$

Thus,

$$
\hat{a}_{n}^{\gamma-1} \hat{L}\left(\hat{a}_{n}\right) \sim \frac{1}{n}, \quad n \rightarrow \infty .
$$

Furthermore, defining $a_{n}:=\inf \left\{k: k^{1-\gamma} L^{-1}(k) \geq n\right\}$, we immediately see that

$$
a_{n}^{\gamma-1} L\left(a_{n}\right) \sim \frac{1}{n}, \quad n \rightarrow \infty
$$

In addition,

$$
\lim _{n \rightarrow \infty} \frac{a_{n}}{\hat{a}_{n}}=\pi_{s}^{1 /(1-\gamma)} .
$$

Consequently, it follows from (2.12)-(2.14), and the fact that $\hat{L}$ is slowly varying that, as $n$ tends to infinity

$$
\hat{L}\left(a_{n}\right) \sim \hat{L}\left(\hat{a}_{n}\right) \sim \pi_{s}^{-1} L\left(a_{n}\right),
$$

and so

$$
\lim _{n \rightarrow \infty} \frac{\hat{L}(n)}{L(n)}=\lim _{n \rightarrow \infty} \frac{\hat{L}\left(a_{\left[n^{1-\gamma} L^{-1}(n)\right]}\right)}{L\left(a_{\left[n^{1-\gamma} L^{-1}(n)\right]}\right)}=\pi_{s}^{-1},
$$

which gives the desired result. 
Define

$$
\psi_{0}(u)=\frac{C_{\alpha}}{2} \int_{\mathbb{R}} \sup _{n \geq 0} \frac{\left(h_{n}(x)\right)_{+}^{\alpha}}{\left(u+\mu_{n}\right)^{\alpha}} \mathrm{d} x+\frac{C_{\alpha}}{2} \int_{\mathbb{R}} \sup _{n \geq 0} \frac{\left(-h_{n}(x)\right)_{+}^{\alpha}}{\left(u+\mu_{n}\right)^{\alpha}} \mathrm{d} x, \quad u>0,
$$

where

$$
C_{\alpha}=\left(\int_{0}^{\infty} x^{-\alpha} \sin x \mathrm{~d} x\right)^{-1}
$$

The following two results can be established via Theorem 2.1 and an argument parallel to that used in [16].

Proposition 2.1. Given (2.3) the following relation holds:

$$
\psi(u) \sim \psi_{0}(u) \quad \text { as } u \rightarrow \infty .
$$

Lemma 2.1. For $s \in \mathbb{Z}$,

$$
m\left(\tau_{s}=k\right)=\pi_{s} \mathrm{P}_{s}\left(\tau_{s} \geq k\right), \quad k=1,2, \ldots,
$$

and

$$
m\left(\tau_{s} \leq n\right) \sim \gamma^{-1} n^{\gamma} L(n) \text { as } n \rightarrow \infty .
$$

The next theorem establishes the main result of this section by showing that the ruin probability $\psi(u)$ may decay very slowly as the initial capital $u$ increases in the setting described above. Note that, unlike Theorem 3.2 of [16], this result is only stated for $\gamma \in(0,1)$, as the solidarity property proved in Theorem 2.1 was shown only for these values of $\gamma$. However, we expect the solidarity property to hold for $\gamma=1$ as well, which in turn should make the following result extendable to this case.

Theorem 2.2. Under assumption (2.3) the following relation holds:

$$
\psi(u) \sim\left(\sum_{s \in A} a_{s} \pi_{s}\right)^{\alpha} A_{\alpha, \gamma} \mu^{\gamma(\alpha-1)-\alpha} u^{-\gamma(\alpha-1)} L^{-(\alpha-1)}(u) \text { as } u \rightarrow \infty,
$$

where

$$
A_{\alpha, \gamma}=\frac{C_{\alpha} \beta(\gamma, \gamma(\alpha-1))}{2} E\left(\sup _{t \geq 1} \frac{t-1}{Z_{1-\gamma}(t)}\right)^{\alpha(1-\gamma)},
$$

and $\beta(\cdot, \cdot)$ is the beta function.

In light of Proposition 2.1, to prove Theorem 2.2 it is enough to show the result for $\psi_{0}(u)$. We start by fixing $s_{0} \in A$.

Lemma 2.2. The following relation holds:

$$
\begin{aligned}
g(u) & :=E_{S_{0}}\left[\sup _{n \geq 0}\left(\frac{\sum_{s \in A} a_{s} N_{n}^{(s)}}{u+n}\right)^{\alpha}\right] \\
& \sim\left(\sum_{s \in A} a_{s} \pi_{s}\right)^{\alpha} E\left(\sup _{t \geq 1} \frac{t-1}{Z_{1-\gamma}(t)}\right)^{\alpha(1-\gamma)} u^{-\gamma \alpha} L^{-\alpha}(u) \text { as } u \rightarrow \infty .
\end{aligned}
$$


Proof. It is easy to see, by (2.5) and the argument given in (2.4), that

$$
\lim _{n \rightarrow \infty} \sum_{s \in A} \frac{a_{s} N_{n}^{(s)}}{N_{n}^{\left(s_{0}\right)}}=\sum_{s \in A} \frac{a_{s} \pi_{s}}{\pi_{s_{0}}}, \quad \mathrm{P}_{s_{0}} \text {-a.s. }
$$

Also, as shown previously, note that

$$
\frac{1}{\pi_{s_{0}}} \eta_{n}^{\left(s_{0}\right)} \Rightarrow Z_{1-\gamma}^{\gamma-1}(1) \quad \text { under } \mathrm{P}_{s_{0}} .
$$

Therefore, Slutsky's theorem now gives

$$
\sum_{s \in A} a_{s} \eta_{n}^{(s)}=\eta_{n}^{\left(s_{0}\right)}\left[\sum_{s \in A} \frac{a_{s} N_{n}^{(s)}}{N_{n}^{\left(s_{0}\right)}}\right] \Rightarrow\left(\sum_{s \in A} a_{s} \pi_{s}\right) Z_{1-\gamma}^{\gamma-1}(1) \quad \text { under } \mathrm{P}_{s_{0}} .
$$

Moreover, by Theorem 2.1 and an argument similar to that of Proposition 3.4 of [19] we can show that all power moments of $\eta_{n}^{(s)}$ converge under $\mathrm{P}_{s}$. In particular, this together with the continuous mapping theorem imply that, for any $\delta>0,\left\{\left(\eta_{n}^{(s)}\right)^{\alpha+\delta}\right\}_{n \geq 1}$ are uniformly integrable under $\mathrm{P}_{s}$. Thus, it follows from Theorem 6.5.1 of [18] that

$$
\sup _{n \geq 1} E_{S}\left[\left(\eta_{n}^{(s)}\right)^{\alpha+\delta}\right]<\infty .
$$

Next, for any $s \neq s_{0}$, observe that by the strong Markov property and Hölder's inequality, we have

$$
\begin{aligned}
\sup _{n \geq 1} E_{S_{0}}\left[\left(\eta_{n}^{(s)}\right)^{\alpha+\delta}\right] & =\sup _{n \geq 1} E_{S_{0}}\left\{\left(n^{\gamma-1} L(n)\right)^{\alpha+\delta} E_{S_{0}}\left[\left(N_{n}^{(s)}\right)^{\alpha+\delta} \mid \tau_{s}\right]\right\} \\
& =\sup _{n \geq 1} E_{S_{0}}\left\{\left(n^{\gamma-1} L(n)\right)^{\alpha+\delta} E_{s}\left[\left(1+N_{n-\tau_{s}}^{(s)}\right)^{\alpha+\delta}\right]\right\} \\
& \leq 2^{\alpha+\delta-1} \sup _{n \geq 1}\left\{\left(n^{\gamma-1} L(n)\right)^{\alpha+\delta}+E_{S}\left[\left(n^{\gamma-1} L(n)\right)^{\alpha+\delta}\left(N_{n-\tau_{s}}^{(s)}\right)^{\alpha+\delta}\right]\right\} \\
& \leq 2^{\alpha+\delta-1}\left\{1+\sup _{n \geq 1} E_{S}\left[\left(\eta_{n}^{(s)}\right)^{\alpha+\delta}\right]\right\} \\
& <\infty .
\end{aligned}
$$

So the 'crystal ball condition' (see, for example, [18, p. 184]) is satisfied; hence, we conclude that $\left\{\left(\eta_{n}^{(s)}\right)^{\alpha}\right\}_{n \geq 1}$ are uniformly integrable under $\mathrm{P}_{S_{0}}$. This, together with the fact that $\left\{\left(\eta_{n}^{\left(s_{0}\right)}\right)^{\alpha}\right\}_{n \geq 1}$ are uniformly integrable under $\mathrm{P}_{s_{0}}$, implies that $\left\{\left(\sum_{s \in A} a_{s} \eta_{n}^{(s)}\right)^{\alpha}\right\}_{n \geq 1}$ are uniformly integrable under $\mathrm{P}_{s_{0}}$ as

$$
\left(\sum_{s \in A} a_{s} \eta_{n}^{(s)}\right)^{\alpha} \leq[\#(A)]^{\alpha-1} \sum_{s \in A} a_{s}^{\alpha}\left(\eta_{n}^{(s)}\right)^{\alpha} .
$$

Then, recalling (2.16) and using the continuous mapping theorem, we see that

$$
\lim _{n \rightarrow \infty} E_{s_{0}}\left(\sum_{s \in A} a_{s} \eta_{n}^{(s)}\right)^{\alpha}=\left(\sum_{s \in A} a_{s} \pi_{s}\right)^{\alpha} E Z_{1-\gamma}^{\alpha(\gamma-1)}(1) .
$$


In particular, we have

$$
E_{s_{0}}\left(\sum_{s \in A} a_{s} N_{n}^{(s)}\right)^{\alpha} \sim\left(\sum_{s \in A} a_{s} \pi_{s}\right)^{\alpha} n^{\alpha(1-\gamma)} L^{-\alpha}(n) E Z_{1-\gamma}^{-\alpha(1-\gamma)}(1) \quad \text { as } n \rightarrow \infty
$$

Now for any $K>0$ consider

$$
g_{K}(u):=E_{S_{0}}\left[\sup _{0 \leq n \leq u K}\left(\frac{\sum_{s \in A} a_{S} N_{n}^{(s)}}{u+n}\right)^{\alpha}\right]
$$

and

$$
g^{K}(u):=E_{s_{0}}\left[\sup _{n>u K}\left(\frac{\sum_{s \in A} a_{s} N_{n}^{(s)}}{u+n}\right)^{\alpha}\right] .
$$

An argument similar to that given in Lemma 3.4 of [16] yields

$$
\lim _{K \uparrow \infty} \limsup _{u \rightarrow \infty} u^{\alpha \gamma} L^{\alpha}(u) g^{K}(u)=0 .
$$

We will next bound $g_{K}(u)$. First, notice it is shown in [16] that as $u$ tends to infinity

$$
\sup _{0 \leq n \leq u K} \frac{u^{\gamma} L(u) N_{n}^{(0)}}{u+n} \Rightarrow \sup _{1 \leq t \leq K+1}\left(\frac{t-1}{Z_{1-\gamma}(t)}\right)^{1-\gamma} \quad \text { under } \mathrm{P}_{0} .
$$

We can use the same argument and Theorem 2.1 to easily see that

$$
\sup _{0 \leq n \leq u K} \frac{u^{\gamma} L(u) N_{n}^{\left(s_{0}\right)}}{\pi_{s_{0}}(u+n)} \Rightarrow \sup _{1 \leq t \leq K+1}\left(\frac{t-1}{Z_{1-\gamma}(t)}\right)^{1-\gamma} \quad \text { under } \mathrm{P}_{s_{0}} .
$$

Next observe that, for $m \geq 1$,

$$
\begin{aligned}
\sup _{0 \leq n \leq u K} & \frac{u^{\gamma} L(u) \sum_{s \in A} a_{s} N_{n}^{(s)}}{u+n} \\
\leq & \sum_{n=0}^{m-1} \frac{u^{\gamma} L(u) \sum_{s \in A} a_{s} N_{n}^{(s)}}{u+n}+\sup _{m \leq n \leq u K} \frac{u^{\gamma} L(u) \sum_{s \in A} a_{s} N_{n}^{(s)}}{u+n} \\
\leq & m^{2} \sum_{s \in A} a_{s} u^{\gamma-1} L(u)+\sup _{m \leq n \leq u K}\left[\frac { u ^ { \gamma } L ( u ) N _ { n } ^ { ( s _ { 0 } ) } } { u + n } \left(\sum_{s \in A} \frac{a_{s} N_{n}^{(s)}}{\left.\left.N_{n}^{\left(s_{0}\right)}\right)\right]}\right.\right. \\
\leq & m^{2} \sum_{s \in A} a_{s} u^{\gamma-1} L(u)+\left[\sup _{m \leq n} \frac{\sum_{s \in A} a_{s} N_{n}^{(s)}}{N_{n}^{\left(s_{0}\right)}}\right]\left[\sup _{0 \leq n \leq u K} \frac{u^{\gamma} L(u) N_{n}^{\left(s_{0}\right)}}{u+n}\right] .
\end{aligned}
$$

Furthermore, for $\varepsilon \in(0,1)$ define

$$
\mathcal{T}_{\varepsilon}:=\inf \left\{k \geq 1: \sum_{s \in A} \frac{a_{s} N_{n}^{(s)}}{N_{n}^{\left(s_{0}\right)}} \geq(1-\varepsilon) \sum_{s \in A} \frac{a_{s} \pi_{s}}{\pi_{s_{0}}}, n \geq k\right\} .
$$


It follows from $(2.15)$ that $\mathcal{T}_{\varepsilon}$ is finite $\mathrm{P}_{s_{0}}$-a.s. Then,

$$
\begin{aligned}
\sup _{0 \leq n \leq u K} & \frac{u^{\gamma} L(u) \sum_{s \in A} a_{s} N_{n}^{(s)}}{u+n} \\
& \geq \sup _{\mathcal{T}_{\varepsilon} \leq n \leq u K}\left[\frac{u^{\gamma} L(u) N_{n}^{\left(s_{0}\right)}}{u+n}\left(\sum_{s \in A} \frac{a_{s} N_{n}^{(s)}}{N_{n}^{\left(s_{0}\right)}}\right)\right] \\
& \geq(1-\varepsilon) \sum_{s \in A} \frac{a_{s} \pi_{s}}{\pi_{s_{0}}}\left[\sup _{\mathcal{T}_{\varepsilon} \leq n \leq u K} \frac{u^{\gamma} L(u) N_{n}^{\left(s_{0}\right)}}{u+n}\right] \\
& \geq(1-\varepsilon) \sum_{s \in A} \frac{a_{s} \pi_{s}}{\pi_{s_{0}}}\left[\sup _{0 \leq n \leq u K} \frac{u^{\gamma} L(u) N_{n}^{\left(s_{0}\right)}}{u+n}-\sup _{0 \leq n \leq \mathcal{T}_{\varepsilon}} \frac{u^{\gamma} L(u) N_{n}^{\left(s_{0}\right)}}{u+n}\right] \\
& \geq(1-\varepsilon) \sum_{s \in A} \frac{a_{s} \pi_{s}}{\pi_{s_{0}}}\left[\sup _{0 \leq n \leq u K} \frac{u^{\gamma} L(u) N_{n}^{\left(s_{0}\right)}}{u+n}-u^{\gamma-1} L(u) \mathcal{T}_{\varepsilon}\right] .
\end{aligned}
$$

Note that, as $u$ goes to infinity,

$$
u^{\gamma-1} L(u) \mathcal{T}_{\varepsilon} \stackrel{\mathrm{P}_{s_{0}}}{\longrightarrow} 0 .
$$

Now recalling (2.18) and Slutsky's theorem, then letting $u$ go to infinity in (2.19) and (2.20), and finally letting $m$ in (2.19) go to infinity and $\varepsilon$ in (2.20) go to zero, we conclude that

$$
\sup _{0 \leq n \leq u K} \frac{u^{\gamma} L(u) \sum_{s \in A} a_{s} N_{n}^{(s)}}{u+n} \Rightarrow\left(\sum_{s \in A} a_{s} \pi_{s}\right) \sup _{1 \leq t \leq K+1}\left(\frac{t-1}{Z_{1-\gamma}(t)}\right)^{1-\gamma} \quad \text { under } \mathrm{P}_{s_{0}} .
$$

Moreover, note that, for any fixed $K>0$,

$$
\left.\left(\sup _{0 \leq n \leq u K} \frac{u^{\gamma} L(u) \sum_{s \in A} a_{s} N_{n}^{(s)}}{u+n}\right)^{\alpha} \leq \text { constant }\right)\left(\sum_{s \in A} a_{s} \eta_{[u K]}^{(s)}\right)^{\alpha},
$$

and the variables on the right-hand side are uniformly integrable under $\mathrm{P}_{s_{0}}$ implying that

$$
\left\{\sup _{0 \leq n \leq u K}\left(\frac{u^{\gamma} L(u) \sum_{s \in A} a_{s} N_{n}^{(s)}}{u+n}\right)^{\alpha}\right\}_{u \geq 0}
$$

are uniformly integrable under $\mathrm{P}_{s_{0}}$. Thus, in particular, applying the continuous mapping theorem we have

$$
\begin{aligned}
\lim _{u \rightarrow \infty} u^{\alpha \gamma} L^{\alpha}(u) g_{K}(u) & =\lim _{u \rightarrow \infty} E_{S_{0}}\left[\sup _{0 \leq n \leq u K}\left(\frac{u^{\gamma} L(u) \sum_{s \in A} a_{s} N_{n}^{(s)}}{u+n}\right)^{\alpha}\right] \\
& =\left(\sum_{s \in A} a_{s} \pi_{s}\right)^{\alpha} E\left(\sup _{1 \leq t \leq K+1} \frac{t-1}{Z_{1-\gamma}(t)}\right)^{\alpha(1-\gamma)} .
\end{aligned}
$$

In addition, it was shown in [16] that, for any $p>0$,

$$
E\left(\sup _{t \geq 1} \frac{t-1}{Z_{1-\gamma}(t)}\right)^{p}<\infty
$$


hence, letting $K$ increase to infinity and recalling (2.17), we have

$$
\lim _{u \rightarrow \infty} u^{\alpha \gamma} L^{\alpha}(u) g(u)=\left(\sum_{s \in A} a_{s} \pi_{s}\right)^{\alpha} E\left(\sup _{t \geq 1} \frac{t-1}{Z_{1-\gamma}(t)}\right)^{\alpha(1-\gamma)}<\infty .
$$

Proof of Theorem 2.2. Note that

$$
\begin{aligned}
\frac{2 \psi_{0}(u)}{C_{\alpha}} & =\int_{E} \sup _{n \geq 0} \frac{\left(\sum_{k=1}^{n} f_{k}(\boldsymbol{y})\right)_{+}^{\alpha}+\left(-\sum_{k=1}^{n} f_{k}(\boldsymbol{y})\right)_{+}^{\alpha}}{(u+n \mu)^{\alpha}} m(\mathrm{~d} \boldsymbol{y}) \\
& =\sum_{i=-\infty}^{\infty} \pi_{i} E_{i}\left[\sup _{n \geq 0}\left(\frac{\sum_{s \in A} a_{s} N_{n}^{(s)}}{u+n \mu}\right)^{\alpha}\right] .
\end{aligned}
$$

For $A=\left\{s_{0}\right\}$, the desired result easily follows from the strong Markov property, Lemma 2.1, Theorem 2.1, Lemma 2.2, and the proof of Theorem 3.2 of [16].

For $A \neq\left\{s_{0}\right\}$ write

$$
\begin{aligned}
\sum_{s \in A} a_{s} N_{n}^{(s)}= & \sum_{s \in A \backslash\left\{s_{0}\right\}} a_{s}\left(G_{s}^{s_{0}} \mathbf{1}_{\left\{\tau_{s} \leq \tau_{s_{0}} \wedge n\right\}} \wedge N_{n}^{(s)}\right) \\
& +\left[\sum_{s \in A} a_{s} N_{n}^{(s)}-\sum_{s \in A \backslash\left\{s_{0}\right\}} a_{s}\left(G_{s}^{s_{0}} \mathbf{1}_{\left\{\tau_{s} \leq \tau_{s_{0}} \wedge n\right\}} \wedge N_{n}^{(s)}\right)\right],
\end{aligned}
$$

where, for any states $s_{1}, s_{2} \in \mathbb{Z}$ and $\boldsymbol{y} \in E$,

$$
G_{s_{1}}^{s_{2}}=G_{s_{1}}^{s_{2}}(\boldsymbol{y}):=\sum_{i=1}^{\tau_{s_{2}}(\boldsymbol{y})} \mathbf{1}_{\left\{y_{i}=s_{1}\right\}}
$$

i.e. $G_{s}^{s_{0}}$ is the number of visits to state $s$ before the first visit to state $s_{0}$. (Note that $G_{s_{1}}^{s_{2}}$ has a geometric distribution under $\mathrm{P}_{s_{1}}$.)

Now we collect some intermediate results, which will be combined at the last stage.

Observe that

$$
\begin{aligned}
& \sum_{i=-\infty}^{\infty} \pi_{i} E_{i}\left[\sup _{n \geq 0}\left(\frac{\sum_{s \in A \backslash\left\{s_{0}\right\}} a_{s}\left(G_{s}^{s_{0}} 1_{\left[\tau_{s} \leq \tau_{s_{0}} \wedge n\right]} \wedge N_{n}^{(s)}\right)}{u+n \mu}\right)^{\alpha}\right] \\
& \leq \sum_{i=-\infty}^{\infty} \pi_{i} E_{i}\left[\left(\sum_{s \in A \backslash\left\{s_{0}\right\}} \sup _{n \geq 0}\left(\frac{a_{s} G_{s}^{s_{0}} \mathbf{1}_{\left\{\tau_{s} \leq \tau_{s_{0}} \wedge n\right\}}}{u+n \mu}\right)^{\alpha}\right]\right. \\
& \quad=\sum_{i=-\infty}^{\infty} \pi_{i} E_{i}\left[\left(\sum_{s \in A \backslash\left\{s_{0}\right\}} \frac{a_{s} G_{s}^{s_{0}}}{u+\tau_{s} \mu}\right)^{\alpha}\right],
\end{aligned}
$$

then it follows from Hölder's inequality and Fubini's theorem that

$$
\sum_{i=-\infty}^{\infty} \pi_{i} E_{i}\left[\left(\sum_{s \in A \backslash\left\{s_{0}\right\}} \frac{a_{s} G_{s}^{s_{0}}}{u+\tau_{s} \mu}\right)^{\alpha}\right] \leq[\#(A)]^{\alpha-1} \sum_{s \in A \backslash\left\{s_{0}\right\}} \sum_{i=-\infty}^{\infty} \pi_{i} E_{i}\left[\left(\frac{a_{s} G_{s}^{s_{0}}}{u+\tau_{s} \mu}\right)^{\alpha}\right]
$$


and by the strong Markov property it follows that

$$
\begin{aligned}
& \sum_{s \in A \backslash\left\{s_{0}\right\}} \sum_{i=-\infty}^{\infty} \pi_{i} E_{i}\left[\left(\frac{a_{s} G_{s}^{s_{0}}}{u+\tau_{s} \mu}\right)^{\alpha}\right] \\
& =\sum_{s \in A \backslash\left\{s_{0}\right\}} a_{s}^{\alpha} \sum_{i=-\infty}^{\infty} \sum_{k=1}^{\infty} \pi_{i} E_{i}\left[\left(\frac{G_{s}^{s_{0}}}{u+\tau_{s} \mu}\right)^{\alpha} \mid \tau_{s}=k\right] \mathrm{P}_{i}\left(\tau_{s}=k\right) \\
& =\sum_{s \in A \backslash\left\{s_{0}\right\}} a_{s}^{\alpha} E_{s}\left[\left(G_{s}^{s_{0}}+1\right)^{\alpha}\right] \sum_{k=1}^{\infty}(u+k \mu)^{-\alpha} m\left(\tau_{s}=k\right) .
\end{aligned}
$$

So by Lemma 2.1, as $u \rightarrow \infty$,

$$
\begin{aligned}
\sum_{i=-\infty}^{\infty} \pi_{i} E_{i}\left[\left(\sum_{s \in A \backslash\left\{s_{0}\right\}} \frac{a_{s} G_{s}^{s_{0}}}{u+\tau_{s} \mu}\right)^{\alpha}\right] & \leq(\text { constant })(u+\mu)^{-(\alpha-1)} \\
& =o\left(u^{-\gamma(\alpha-1)} L^{-(\alpha-1)}(u)\right) .
\end{aligned}
$$

Additionally, by the strong Markov property, Lemma 2.1, Theorem 2.1, Lemma 2.2, and Lemma 3.6 of [16], we have

$$
\begin{aligned}
& \sum_{i=-\infty}^{\infty} \pi_{i} E_{i}\left[\sup _{n \geq \tau_{s_{0}}}\left(\frac{\sum_{s \in A} a_{s} N_{n}^{(s)}-\sum_{s \in A \backslash\left\{s_{0}\right\}} a_{s} G_{s}^{s_{0}}}{u+n \mu}\right)^{\alpha}\right] \\
& \quad=\sum_{k=1}^{\infty} m\left(\tau_{s_{0}}=k\right) E_{s_{0}}\left[\sup _{n \geq 0}\left(\frac{\sum_{s \in A} a_{s} N_{n}^{(s)}}{u+(n+k) \mu}\right)^{\alpha}\right] \\
& =\mu^{-\alpha} \sum_{k=1}^{\infty} \mathrm{P}_{0}\left(\tau_{0} \geq k\right) g\left(k+\frac{u}{\mu}\right) \\
& \sim \frac{2\left(\sum_{s \in A} a_{s} \pi_{s}\right)^{\alpha} A_{\alpha, \gamma}}{C_{\alpha}} \mu^{\gamma(\alpha-1)-\alpha} u^{-\gamma(\alpha-1)} L^{-(\alpha-1)}(u) \quad \text { as } u \rightarrow \infty
\end{aligned}
$$

Furthermore, notice that $G_{s}^{s_{0}}=G_{s}^{s_{0}} \mathbf{1}_{\left\{\tau_{s}<\tau_{s_{0}}\right\}} \leq G_{s}^{s_{0}}\left(u+\tau_{s_{0}} \mu\right) /\left(u+\tau_{s} \mu\right)$. Consequently, by (2.25) we have

$$
\sum_{i=-\infty}^{\infty} \pi_{i} E_{i}\left[\left(\frac{\sum_{s \in A \backslash\left\{s_{0}\right\}} a_{s} G_{s}^{s_{0}}}{u+\tau_{s_{0}} \mu}\right)^{\alpha}\right]=o\left(u^{-\gamma(\alpha-1)} L^{-(\alpha-1)}(u)\right) \quad \text { as } u \rightarrow \infty .
$$

Now, for any $M>0$,

$$
\begin{gathered}
\sum_{i=-\infty}^{\infty} \pi_{i} E_{i}\left(\sup _{n \geq \tau_{s_{0}}} \frac{\left(\sum_{s \in A \backslash\left\{s_{0}\right\}} a_{s} G_{s}^{s_{0}}\right)\left(\sum_{s \in A} a_{s} N_{n}^{(s)}-\sum_{s \in A \backslash\left\{s_{0}\right\}} a_{s} G_{s}^{s_{0}}\right)^{\alpha-1}}{(u+n \mu)^{\alpha}}\right) \\
\leq \sum_{i=-\infty}^{\infty} \pi_{i} E_{i}\left(\sup _{n \geq \tau_{s_{0}}} \frac{\left(\sum_{s \in A \backslash\left\{s_{0}\right\}} a_{s} G_{s}^{s_{0}}\right) \mathbf{1}_{\left\{\sum_{s \in A} a_{s} N_{n}^{(s)}<(M+1) \sum_{\left.s \in A \backslash s_{0}\right\}} a_{s} G_{s}^{s_{0}}\right\}}}{\left(\sum_{s \in A} a_{s} N_{n}^{(s)}\right)^{1-\alpha}(u+n \mu)^{\alpha}}\right) \\
\quad+\frac{1}{M} \sum_{i=-\infty}^{\infty} \pi_{i} E_{i}\left(\sup _{n \geq \tau_{s_{0}}}\left(\frac{\sum_{s \in A} a_{s} N_{n}^{(s)}-\sum_{s \in A \backslash\left\{s_{0}\right\}} a_{s} G_{s}^{s_{0}}}{u+n \mu}\right)^{\alpha}\right),
\end{gathered}
$$


and it follows from (2.26) and (2.27) that, as $u \rightarrow \infty$,

$$
\begin{gathered}
\sum_{i=-\infty}^{\infty} \pi_{i} E_{i}\left(\sup _{n \geq \tau_{s_{0}}} \frac{\left(\sum_{s \in A \backslash\left\{s_{0}\right\}} a_{s} G_{s}^{s_{0}}\right)\left(\sum_{s \in A} a_{s} N_{n}^{(s)}-\sum_{s \in A \backslash\left\{s_{0}\right\}} a_{s} G_{s}^{s_{0}}\right)^{\alpha-1}}{(u+n \mu)^{\alpha}}\right) \\
\leq(M+1)^{\alpha-1} \sum_{i=-\infty}^{\infty} \pi_{i} E_{i}\left[\left(\frac{\sum_{s \in A \backslash\left\{s_{0}\right\}} a_{s} G_{s}^{s_{0}}}{u+\tau_{s_{0}} \mu}\right)^{\alpha}\right] \\
\quad+\frac{1}{M} \mu^{-\alpha} \sum_{k=1}^{\infty} \mathrm{P}_{0}\left(\tau_{0} \geq k\right) g\left(k+\frac{u}{\mu}\right) \\
\sim M^{-1} \frac{2\left(\sum_{s \in A} a_{s} \pi_{s}\right)^{\alpha} A_{\alpha, \gamma}}{C_{\alpha}} \mu^{\gamma(\alpha-1)-\alpha} u^{-\gamma(\alpha-1)} L^{-(\alpha-1)}(u) .
\end{gathered}
$$

However, as $M>0$ is arbitrary we conclude that

$$
\begin{aligned}
& \sum_{i=-\infty}^{\infty} \pi_{i} E_{i}\left(\sup _{n \geq \tau_{s_{0}}} \frac{\left(\sum_{s \in A \backslash\left\{s_{0}\right\}} a_{s} G_{s}^{s_{0}}\right)\left(\sum_{s \in A} a_{s} N_{n}^{(s)}-\sum_{s \in A \backslash\left\{s_{0}\right\}} a_{s} G_{s}^{s_{0}}\right)^{\alpha-1}}{(u+n \mu)^{\alpha}}\right) \\
& =o\left(u^{-\gamma(\alpha-1)} L^{-(\alpha-1)}(u)\right) \quad \text { as } u \rightarrow \infty .
\end{aligned}
$$

Lastly, observe that

$$
\begin{aligned}
& n<\tau_{s_{0}} \Longrightarrow \sum_{s \in A} a_{s} N_{n}^{(s)}-\sum_{s \in A \backslash\left\{s_{0}\right\}} a_{s}\left(G_{s}^{s_{0}} \mathbf{1}_{\left\{\tau_{s} \leq \tau_{s_{0}} \wedge n\right\}} \wedge N_{n}^{(s)}\right)=0, \\
& n \geq \tau_{s_{0}} \Longrightarrow G_{S}^{s_{0}} \mathbf{1}_{\left\{\tau_{s} \leq \tau_{s_{0}} \wedge n\right\}} \wedge N_{n}^{(s)}=G_{s}^{s_{0}},
\end{aligned}
$$

and notice by the convexity of the function $c(x)=x^{\alpha}$ for $x \geq 0$, for any $x_{0}, y_{0} \geq 0$, we have

$$
\begin{aligned}
\frac{\left(x_{0}+y_{0}\right)^{\alpha}-x_{0}^{\alpha}}{y_{0}} & \leq \alpha\left(x_{0}+y_{0}\right)^{\alpha-1} \leq \alpha\left(x_{0}^{\alpha-1}+y_{0}^{\alpha-1}\right) \\
& \Longrightarrow\left(x_{0}+y_{0}\right)^{\alpha} \leq x_{0}^{\alpha}+\alpha x_{0}^{\alpha-1} y_{0}+\alpha y_{0}^{\alpha}
\end{aligned}
$$

So it follows from (2.21), (2.22), (2.29), and (2.30) that

$$
\begin{aligned}
\frac{2 \psi_{0}(u)}{C_{\alpha}} \leq & \sum_{i=-\infty}^{\infty} \pi_{i} E_{i}\left[\sup _{n \geq \tau_{s_{0}}}\left(\frac{\sum_{s \in A} a_{s} N_{n}^{(s)}-\sum_{s \in A \backslash\left\{s_{0}\right\}} a_{s} G_{s}^{s_{0}}}{u+n \mu}\right)^{\alpha}\right] \\
& +\alpha \sum_{i=-\infty}^{\infty} \pi_{i} E_{i}\left[\sup _{n \geq \tau_{s_{0}}} \frac{\left(\sum_{s \in A \backslash\left\{s_{0}\right\}} a_{s} G_{s}^{s_{0}}\right)\left(\sum_{s \in A} a_{s} N_{n}^{(s)}-\sum_{s \in A \backslash\left\{s_{0}\right\}} a_{s} G_{s}^{s_{0}}\right)^{\alpha-1}}{(u+n \mu)^{\alpha}}\right] \\
& +\alpha \sum_{i=-\infty}^{\infty} \pi_{i} E_{i}\left[\sup _{n \geq 0}\left(\frac{\sum_{s \in A \backslash\left\{s_{0}\right\}} a_{s}\left(G_{s}^{s_{0}} \mathbf{1}_{\left\{\tau_{s} \leq \tau_{s_{0}} \wedge n\right\}} \wedge N_{n}^{(s)}\right)}{u+n \mu}\right)^{\alpha}\right] .
\end{aligned}
$$

Finally, the desired result follows from (2.23), (2.25), (2.26), (2.28), and (2.31). 


\section{A continuous time stationary $S \alpha S$ process associated with a conservative flow}

In this section we consider a class of continuous-time claim processes $\boldsymbol{X}$ generated by a conservative flow. The construction of the class of such processes is due to Samorodnitsky [22]. Samorodnitsky constructed a $\mathrm{S} \alpha \mathrm{S}$ random measure $M(\cdot)$ using a standard $H$-fractional Brownian motion, a centered, stationary increment Gaussian process, with self-similarity exponent $H \in(0,1)$. (See [23, pp. 318-339] or [8, pp. 43-55] for details on this process.) He then used $M$ to describe a $\mathrm{S} \alpha \mathrm{S}$ process $\boldsymbol{X}$ represented by a stochastic integral, and showed that this process is generated by a conservative flow for a certain class of kernels in the integral representation.

In this section we look at the Brownian motion case $\left(H=\frac{1}{2}\right)$, and we pick a fairly simple kernel in this class to show that even then (at least in the context of risk theory) the process is long-range dependent.

The continuous-time model in the insurance is of interest as an approximation in the presence of high-frequency claims which are irregularly spaced. The model can also be applied in the context of fluid queues and storage/dam processes. We continue to use the insurance risk theory language, however informally, and we give further details below.

\subsection{Setup and preliminaries}

Let $\boldsymbol{B}=\{B(t), t \in \mathbb{R}\}$ be a standard Brownian motion. Pick $E=C(-\infty, \infty)$, and let $m$ be a $\sigma$-finite cylindrical measure on $E$ defined by

$$
m(A)=\int_{\mathbb{R}} \mathrm{P}(\boldsymbol{B} \in A-y) \mathrm{d} y, \quad A \text { a cylindrical set, }
$$

i.e. $m$ is the (infinite) law of the Brownian motion shifted according to the Lebesgue measure on $\mathbb{R}$. Define

$$
\varphi(x):=(1-|x|) \mathbf{1}_{\{(1-|x|) \in[0,1]\}}, \quad x \in \mathbb{R} .
$$

Note that $\varphi: \mathbb{R} \mapsto[0, \infty)$ is Hölder continuous with exponent one, even, nonincreasing on $[0, \infty)$, and $\varphi \in L^{\alpha}(\mathbb{R}, \mathcal{B}, \lambda)$. Clearly, the Hölder function

$$
H(x)=\sup _{x \leq s<t} \frac{\varphi(s)-\varphi(t)}{t-s}, \quad x \geq 0
$$

also belongs to $L^{\alpha}(\mathbb{R}, \mathcal{B}, \lambda)$. Furthermore, define

$$
X(t)=\int_{E} \varphi\left(x_{t}\right) M(\mathrm{~d} \boldsymbol{x}), \quad t \in \mathbb{R}, \boldsymbol{x}=\left(x_{s}, s \in \mathbb{R}\right),
$$

where $M$ is a $\mathrm{S} \alpha \mathrm{S}$ random measure on $E$ with control measure $m$. It is shown in [22] that the process $\boldsymbol{X}=\{X(t), t \in \mathbb{R}\}$ is a well defined stationary $S \alpha S$ process, and is generated by a conservative flow.

Now let the process $S=\{S(t), t \geq 0\}$ be given by

$$
S(t):=\int_{0}^{t} X(s) \mathrm{d} s, \quad t \geq 0 .
$$


Note that, for any $T \in(0, \infty)$,

$$
\begin{aligned}
\int_{0}^{T}\left(\int_{E} \varphi\left(x_{S}\right)^{\alpha} m(\mathrm{~d} \boldsymbol{x})\right)^{1 / \alpha} \mathrm{d} s & \leq T\left(\sup _{0 \leq s \leq T} \int_{E} \varphi\left(x_{s}\right)^{\alpha} m(\mathrm{~d} \boldsymbol{x})\right)^{1 / \alpha} \\
& \leq T\left(\int_{E} \sup _{0 \leq s \leq T} \varphi\left(x_{s}\right)^{\alpha} m(\mathrm{~d} \boldsymbol{x})\right)^{1 / \alpha} .
\end{aligned}
$$

However, it was shown in [22] that

$$
b(T):=\left(\int_{E} \sup _{0 \leq s \leq T} \varphi\left(x_{s}\right)^{\alpha} m(\mathrm{~d} \boldsymbol{x})\right)^{1 / \alpha}
$$

is finite. Thus, it follows from Theorem 11.3.2 of [23] that

$$
\int_{0}^{T}|X(s)| \mathrm{d} s<\infty \text { a.s. }
$$

In particular, the process $\{S(t), t \in[0, T]\}$ is well-defined for any $T \in(0, \infty)$ and, hence, $S$ is also well defined.

Next, let

$$
h_{t}(\boldsymbol{x}):=\int_{0}^{t} \varphi\left(x_{s}\right) \mathrm{d} s .
$$

It follows from Theorem 11.4.1 of [23] that

$$
S(t)=\int_{E} h_{t}(\boldsymbol{x}) M(\mathrm{~d} \boldsymbol{x}) \quad \text { a.s., } \quad t \geq 0 .
$$

Now, with $\mathbb{T}=\mathbb{R}_{+}$, the ruin probability given in (1.1) becomes

$$
\psi(u)=\mathrm{P}\left(\sup _{t \geq 0}(S(t)-\mu t)>u\right), \quad u>0 .
$$

Lastly, for $u>0$, let

$$
\psi_{0}(u):=\frac{C_{\alpha}}{2} \int_{\mathbb{R}} \sup _{t \geq 0}\left(\frac{h_{t}(x)}{u+t \mu}\right)^{\alpha} \mathrm{d} x=\frac{C_{\alpha}}{2} \int_{\mathbb{R}} E\left[\sup _{t \geq 0}\left(\frac{\int_{0}^{t} \varphi(B(s)+y) \mathrm{d} s}{u+t \mu}\right)^{\alpha}\right] \mathrm{d} y,
$$

where $\mu>0$ is the deterministic drift rate and $C_{\alpha}=\left(\int_{0}^{\infty} x^{-\alpha} \sin x \mathrm{~d} x\right)^{-1}$.

\subsection{Asymptotic behavior of the ruin probability}

We first prove the asymptotic equivalence of the ruin probability, $\psi(u)$, and $\psi_{0}(u)$ as $u$ goes to infinity.

Proposition 3.1. In the above setting,

$$
\psi(u) \sim \psi_{0}(u) \quad \text { as } u \rightarrow \infty
$$


To prove Proposition 3.1, we need the following two lemmas.

Lemma 3.1. The following relation holds in the setting described above:

$$
\left\|h_{t}(\cdot)\right\|_{L^{\alpha}(E, \mathcal{E}, m)}=O\left(t^{(\alpha+1) / 2 \alpha}\right), \quad t \rightarrow \infty .
$$

Proof. Let $\{l(x, t), x \in \mathbb{R}, t \geq 0\}$ be a jointly continuous local time process of $\boldsymbol{B}$ (see [8, p. 52] for a brief definition or [3] for details). As an immediate consequence of the selfsimilarity of the Brownian motion, local time process has the following scaling property: for any $c>0$,

$$
\left\{l\left(c^{1 / 2} x, c t\right), x \in \mathbb{R}, t \geq 0\right\} \stackrel{\mathrm{D}}{=}\left\{c^{1 / 2} l(x, t), x \in \mathbb{R}, t \geq 0\right\} .
$$

Moreover, all moments of $l(x, t)$ are finite, and are uniformly bounded in all real $x$ and all real $t$ in a compact set. (See, for instance, [5] for details.)

Now, by Hölder's inequality and Fubini's theorem, we have

$$
\begin{aligned}
\left\|h_{t}(\cdot)\right\|_{L^{\alpha}(E, \mathcal{E}, m)}^{\alpha} & =\int_{E} h_{t}^{\alpha}(\boldsymbol{x}) m(\mathrm{~d} \boldsymbol{x}) \\
& =\int_{\mathbb{R}} E\left[\left(\int_{0}^{t} \varphi(B(s)+y) \mathrm{d} s\right)^{\alpha}\right] \mathrm{d} y \\
& =\int_{\mathbb{R}} E\left[\left(\int_{\mathbb{R}} \varphi(x+y) l(x, t) \mathrm{d} x\right)^{\alpha}\right] \mathrm{d} y \\
& \leq \int_{\mathbb{R}} E\left[\left(\int_{-1-y}^{1-y} l(x, t) \mathrm{d} x\right)^{\alpha}\right] \mathrm{d} y \\
& \leq 2^{\alpha-1} \int_{\mathbb{R}} E\left(\int_{-1-y}^{1-y} l^{\alpha}(x, t) \mathrm{d} x\right) \mathrm{d} y \\
& =2^{\alpha} \int_{\mathbb{R}} E\left[l^{\alpha}(x, t)\right] \mathrm{d} x,
\end{aligned}
$$

and by (3.1) we have

$$
\begin{aligned}
\int_{\mathbb{R}} E\left[l^{\alpha}(x, t)\right] \mathrm{d} x & =t^{\alpha / 2} \int_{\mathbb{R}} E\left[l^{\alpha}\left(\frac{x}{\sqrt{t}}, 1\right)\right] \mathrm{d} x \\
& =t^{\alpha / 2} \int_{\mathbb{R}} E\left[l^{\alpha}\left(\frac{x}{\sqrt{t}}, 1\right) \mathbf{1}_{\left\{\sup _{0 \leq s \leq 1}|B(s)| \geq|x / \sqrt{t}|\right\}}\right] \mathrm{d} x \\
& \leq t^{\alpha / 2} \int_{\mathbb{R}}\left[E\left[l^{2}\left(\frac{x}{\sqrt{t}}, 1\right)\right]\right]^{\alpha / 2}\left[\mathrm{P}\left(\sup _{0 \leq s \leq 1}|B(s)| \geq\left|\frac{x}{\sqrt{t}}\right|\right)\right]^{(2-\alpha) / 2} \mathrm{~d} x \\
& \leq(\text { constant }) t^{(\alpha+1) / 2} \int_{\mathbb{R}}\left[\mathrm{P}\left(\sup _{0 \leq s \leq 1}|B(s)| \geq|x|\right)\right]^{(2-\alpha) / 2} \mathrm{~d} x .
\end{aligned}
$$

(The last inequality is due to the fact that the moments of the local time are uniformly bounded.) Finally, the desired result follows by observing that

$$
\int_{\mathbb{R}}\left[\mathrm{P}\left(\sup _{0 \leq s \leq 1}|B(s)| \geq|x|\right)\right]^{(2-\alpha) / 2} \mathrm{~d} x<\infty
$$

as the supremum of a bounded Gaussian process has Gaussian-like tails. (See, for instance, [1].) 
Lemma 3.2. There exists an $\tilde{\varepsilon} \in(0,1)$ such that the process $\tilde{\boldsymbol{Y}}=(\tilde{Y}(t), t \geq 0)$ defined by

$$
\tilde{Y}(t):=(t+1)^{\tilde{\varepsilon}-1} S(t), \quad t \geq 0,
$$

is a.s. bounded.

Proof. It follows from Proposition 7.4 of [4] and Lemma 3.1 that there exists an $\varepsilon_{0}>0$ such that the process

$$
\left((n+1)^{\varepsilon_{0}-1} S(n), n=0,1,2, \ldots\right)
$$

is a.s. bounded.

Furthermore, note by the stationarity of $\boldsymbol{X}$, for any $\tilde{\varepsilon} \in(0,1)$,

$$
\begin{aligned}
\mathrm{P}\left(\sup _{n=0,1,2, \ldots} \frac{\sup _{n \leq t \leq n+1}|S(t)-S(n)|}{(n+1)^{1-\tilde{\varepsilon}}} \geq \lambda\right) \\
\quad \leq \sum_{n=0}^{\infty} \mathrm{P}\left(\sup _{n \leq t \leq n+1}|S(t)-S(n)| \geq \lambda(n+1)^{1-\tilde{\varepsilon}}\right) \\
\quad=\sum_{n=0}^{\infty} \mathrm{P}\left(\sup _{0 \leq t \leq 1}|S(t)| \geq \lambda(n+1)^{1-\tilde{\varepsilon}}\right) \\
\quad \leq \sum_{n=0}^{\infty} \mathrm{P}\left(\sup _{0 \leq s \leq 1}|X(s)| \geq \lambda(n+1)^{1-\tilde{\varepsilon}}\right) .
\end{aligned}
$$

Also, it was shown in [22] that the process $\boldsymbol{X}$ is a.s. sample continuous. Consequently, $(X(s), s \in[0,1])$ is a.s. bounded. Then it follows from Theorem 10.5.1 of [23] that

$$
\sum_{n=0}^{\infty} \mathrm{P}\left(\sup _{0 \leq s \leq 1}|X(s)| \geq \lambda(n+1)^{1-\tilde{\varepsilon}}\right) \leq C \sum_{n=0}^{\infty}\left[\lambda(n+1)^{1-\tilde{\varepsilon}}\right]^{-\alpha},
$$

for some positive constant $C$. Hence, for any $\tilde{\varepsilon}<\left(1-\alpha^{-1}\right)$, we see that

$$
\lim _{\lambda \rightarrow \infty} \mathrm{P}\left(\sup _{n=0,1,2, \ldots} \frac{\sup _{n \leq t \leq n+1}|S(t)-S(n)|}{(n+1)^{1-\tilde{\varepsilon}}} \geq \lambda\right)=0 .
$$

Consequently, for any such $\tilde{\varepsilon}$, it follows from the monotone convergence theorem that the process

$$
\left((n+1)^{\tilde{\varepsilon}-1} \sup _{n \leq t \leq n+1}|S(t)-S(n)|, n=0,1,2, \ldots\right)
$$

is a.s. bounded.

The desired result follows by picking $\tilde{\varepsilon} \in\left(0, \min \left\{\varepsilon_{0},\left(1-\alpha^{-1}\right)\right\}\right)$ and observing that

$$
\sup _{t \geq 0}|\tilde{Y}(t)| \leq \sup _{n=0,1,2, \ldots}(n+1)^{\tilde{\varepsilon}-1}|S(n)|+\sup _{n=0,1,2, \ldots}(n+1)^{\tilde{\varepsilon}-1} \sup _{n \leq t \leq n+1}|S(t)-S(n)| .
$$

Proof of Proposition 3.1. Pick $\tilde{\varepsilon}>0$ such that $\tilde{\boldsymbol{Y}}$ is a.s. bounded and define a process $\boldsymbol{Y}=(Y(t), t \geq 0)$ by

$$
Y(t):=\frac{[\log (t \mu+2)]^{1+\varepsilon}}{t \mu+2} S(t), \quad t \geq 0 .
$$


Note that, for any $\varepsilon>0$,

$$
\frac{[\log (t \mu+2)]^{1+\varepsilon}}{t \mu+2}=o\left((t+1)^{\tilde{\varepsilon}-1}\right) \quad \text { as } t \rightarrow \infty .
$$

Then, as $\tilde{\varepsilon}>0$ is picked such that $\tilde{\boldsymbol{Y}}$ is a.s. bounded, we see that, for any $\varepsilon>0, \boldsymbol{Y}$ is a.s. bounded. Now, the proposition follows from Theorem 4.1 and Remark 4.2 of [4].

What follows is the key step for the proof of the main theorem of this section.

Lemma 3.3. For any $y \in \mathbb{R}$, as $u \rightarrow \infty$, the following relationship holds:

$$
g(u, y):=E\left[\sup _{t \geq 0}\left(\frac{\int_{0}^{t} \varphi(B(s)+y) \mathrm{d} s}{u+t}\right)^{\alpha}\right] \sim u^{-\alpha / 2} E\left[\sup _{t \geq 0}\left(\frac{l(0, t)}{1+t}\right)^{\alpha}\right] .
$$

Proof. Fix $y \in \mathbb{R}$. For $K>0$ start by defining

$$
g^{K}(u, y):=E\left[\sup _{t \geq u K}\left(\frac{\int_{0}^{t} \varphi(B(s)+y) \mathrm{d} s}{u+t}\right)^{\alpha}\right],
$$

and

$$
g_{K}(u, y):=E\left[\sup _{0 \leq t \leq u K}\left(\frac{\int_{0}^{t} \varphi(B(s)+y) \mathrm{d} s}{u+t}\right)^{\alpha}\right] .
$$

Observe, by Hölder's inequality and Fubini's theorem, that

$$
\begin{aligned}
g^{K}(u, y) & \leq \sum_{j=1}^{\infty} E\left[\sup _{u K 2^{j-1} \leq t \leq u K 2^{j}}\left(\frac{\int_{0}^{t} \varphi(B(s)+y) \mathrm{d} s}{u+t}\right)^{\alpha}\right] \\
& \leq u^{-\alpha} \sum_{j=1}^{\infty} E\left(\frac{\int_{0}^{u K 2^{j}} \varphi(B(s)+y) \mathrm{d} s}{1+K 2^{j-1}}\right)^{\alpha} \\
& \leq 2^{\alpha} u^{-\alpha} \sum_{j=1}^{\infty} E\left(\frac{\int_{-1-y}^{1-y} l\left(x, u K 2^{j}\right) \mathrm{d} x}{K 2^{j}}\right)^{\alpha}
\end{aligned}
$$

and, by (3.1) and Hölder's inequality,

$$
\begin{aligned}
& u^{-\alpha} \sum_{j=1}^{\infty} E\left(\frac{\int_{-1-y}^{1-y} l\left(x, u K 2^{j}\right) \mathrm{d} x}{K 2^{j}}\right)^{\alpha} \\
& \quad=u^{-\alpha} \sum_{j=1}^{\infty} E\left[\frac{\sqrt{u K 2^{j}} \int_{-1-y}^{1-y} l\left(x / \sqrt{u K 2^{j}}, 1\right) \mathrm{d} x}{K 2^{j}}\right]^{\alpha} \\
& \quad \leq 2^{\alpha-1} u^{-\alpha / 2} K^{-\alpha / 2} \sum_{j=1}^{\infty} 2^{-j \alpha / 2} \int_{-1-y}^{1-y} E\left[l^{\alpha}\left(\frac{x}{\sqrt{u K 2^{j}}}, 1\right)\right] \mathrm{d} x
\end{aligned}
$$

Then, it follows from the fact that the local time has moments of all orders finite and uniformly bounded in all real $x$,

$$
\lim _{K \uparrow \infty} \limsup _{u \rightarrow \infty} u^{\alpha / 2} g^{K}(u, y)=0 .
$$


Next we will investigate $g_{K}(u, y)$. Start by noting that

$$
\begin{aligned}
\sup _{0 \leq t \leq u K} \frac{\sqrt{u} \int_{0}^{t} \varphi(B(s)+y) \mathrm{d} s}{u+t} & \leq u^{-1 / 2} \int_{0}^{u K} \varphi(B(s)+y) \mathrm{d} s \\
& \leq u^{-1 / 2} \int_{0}^{u K} \mathbf{1}_{\{B(s) \in[-1-y, 1-y]\}} \mathrm{d} s \\
& =u^{-1 / 2} \int_{-1-y}^{1-y} l(x, u K) \mathrm{d} x,
\end{aligned}
$$

and it follows from Hölder's inequality that, for any $\delta>0$,

$$
\left(\sup _{0 \leq t \leq u K} \frac{\sqrt{u} \int_{0}^{t} \varphi(B(s)+y) \mathrm{d} s}{u+t}\right)^{\alpha+\delta} \leq \frac{2^{\alpha+\delta-1}}{u^{(\alpha+\delta) / 2}} \int_{-1-y}^{1-y} l^{\alpha+\delta}(x, u K) \mathrm{d} x .
$$

Consequently, by Fubini's theorem and (3.1) we have

$$
\begin{aligned}
\sup _{u>0} E\left(\left|\sup _{0 \leq t \leq u K} \frac{\sqrt{u} \int_{0}^{t} \varphi(B(s)+y) \mathrm{d} s}{u+t}\right|^{\alpha+\delta}\right) & \leq \sup _{u>0} \frac{2^{\alpha+\delta-1}}{u^{(\alpha+\delta) / 2}} E\left(\int_{-1-y}^{1-y} l^{\alpha+\delta}(x, u K) \mathrm{d} x\right) \\
& =2^{\alpha+\delta-1} \sup _{u>0} \int_{-1-y}^{1-y} E\left[l^{\alpha+\delta}\left(\frac{x}{\sqrt{u}}, K\right)\right] \mathrm{d} x .
\end{aligned}
$$

However, local time $l(x, t)$ has moments of all orders finite and uniformly bounded in all real $x$ and all $t$ in a compact set. Thus, we conclude that

$$
\sup _{u>0} E\left(\left|\sup _{0 \leq t \leq u K} \frac{\sqrt{u} \int_{0}^{t} \varphi(B(s)+y) \mathrm{d} s}{u+t}\right|^{\alpha+\delta}\right)<\infty,
$$

and it follows from the 'crystal ball condition' (cf. [18, p. 184]) that, for any $y \in \mathbb{R}$, the family

$$
\left\{\left(\sup _{0 \leq t \leq u K} \frac{\sqrt{u} \int_{0}^{t} \varphi(B(s)+y) \mathrm{d} s}{u+t}\right)^{\alpha}\right\}_{u>0}
$$

is uniformly integrable.

Next, observe that

$$
\left(u^{-1 / 2} \int_{0}^{u t} \varphi(B(s)+y) \mathrm{d} s, t \geq 0\right) \Rightarrow(l(0, t), t \geq 0),
$$

in $C[0, \infty)$ as $u \rightarrow \infty$. (See, for instance, [8, p. 52] for details.) Thus, for any continuity point $z \geq 0$ of the distribution of $\sup _{0 \leq v \leq K}[l(0, v) /(1+v)]$, as $u \rightarrow \infty$,

$$
\begin{aligned}
\mathrm{P}\left(\sup _{0 \leq t \leq u K} \frac{u^{1 / 2} \int_{0}^{t} \varphi(B(s)+y) \mathrm{d} s}{u+t} \geq z\right) \\
\quad=\mathrm{P}\left(u^{-1 / 2} \int_{0}^{u v} \varphi(B(s)+y) \mathrm{d} s \geq(1+v) z \text { for some } v \leq K\right) \\
\quad \sim \mathrm{P}(l(0, v) \geq(1+v) z \text { for some } v \leq K) \\
\quad=\mathrm{P}\left(\sup _{0 \leq v \leq K} \frac{l(0, v)}{1+v} \geq z\right) .
\end{aligned}
$$


Hence, we conclude that, as $u \rightarrow \infty$,

$$
\sup _{0 \leq t \leq u K} \frac{u^{1 / 2} \int_{0}^{t} \varphi(B(s)+y) \mathrm{d} s}{u+t} \Rightarrow \sup _{0 \leq t \leq K} \frac{l(0, t)}{1+t},
$$

and therefore, by the continuous mapping theorem,

$$
\left(\sup _{0 \leq t \leq u K} \frac{u^{1 / 2} \int_{0}^{t} \varphi(B(s)+y) \mathrm{d} s}{u+t}\right)^{\alpha} \Rightarrow\left(\sup _{0 \leq t \leq K} \frac{l(0, t)}{1+t}\right)^{\alpha} .
$$

Now, recalling the uniform integrability, Theorem 6.6.1 of [18] implies that

$$
\lim _{u \rightarrow \infty} u^{\alpha / 2} g_{K}(u, y)=E\left[\left(\sup _{0 \leq t \leq K} \frac{l(0, t)}{1+t}\right)^{\alpha}\right]
$$

and, thus,

$$
\lim _{K \uparrow \infty} \lim _{u \rightarrow \infty} u^{\alpha / 2} g_{K}(u, y)=E\left[\left(\sup _{t \geq 0} \frac{l(0, t)}{1+t}\right)^{\alpha}\right] .
$$

Lastly, recalling (3.2) we have

$$
\lim _{u \rightarrow \infty} u^{\alpha / 2} g(u, y)=E\left[\left(\sup _{t \geq 0} \frac{l(0, t)}{1+t}\right)^{\alpha}\right] .
$$

Now we state our theorem.

Theorem 3.1. The following relation holds:

$$
\psi(u) \sim \frac{C_{\alpha}}{\sqrt{2 \pi}} E\left[\sup _{t \geq 0}\left(\frac{l(0, t)}{1+t}\right)^{\alpha}\right] \beta\left(\frac{1}{2}, \frac{\alpha-1}{2}\right) \mu^{-(1 / 2)(\alpha+1)} u^{(1 / 2)(1-\alpha)}, \quad u \rightarrow \infty,
$$

where $\beta(\cdot, \cdot)$ is the beta function.

To prove Theorem 3.1, we need the following lemma.

Lemma 3.4. For $y \in \mathbb{R}$, let

$$
I(u, y):=\int_{0}^{\infty} v^{-1 / 2} g(u+v, y) \mathrm{d} v .
$$

Then, as $u \rightarrow \infty$,

$$
I(u, y) \sim u^{(1 / 2)(1-\alpha)} E\left[\sup _{t \geq 0}\left(\frac{l(0, t)}{1+t}\right)^{\alpha}\right] \beta\left(\frac{1}{2}, \frac{\alpha-1}{2}\right) .
$$

Proof. Choose $K>0$. Define

$$
I_{1}(u, y):=\int_{u K}^{\infty} v^{-1 / 2} g(u+v, y) \mathrm{d} v \quad \text { and } \quad I_{2}(u, y):=\int_{0}^{u K} v^{-1 / 2} g(u+v, y) \mathrm{d} v .
$$

Note, by the monotonicity of $g$, that

$$
I_{1}(u, y) \leq \int_{u K}^{\infty} v^{-1 / 2} g(v, y) \mathrm{d} v .
$$


Fix $\varepsilon>0$. Then it follows from Lemma 3.3 that, for sufficiently large $u$,

$$
I_{1}(u, y) \leq(1+\varepsilon) E\left[\sup _{t \geq 0}\left(\frac{l(0, t)}{1+t}\right)^{\alpha}\right] \int_{u K}^{\infty} v^{-(1+\alpha) / 2} \mathrm{~d} v
$$

and, hence,

$$
\lim _{K \uparrow \infty} \limsup _{u \rightarrow \infty} u^{(1 / 2)(\alpha-1)} I_{1}(u, y)=0 .
$$

Also, by Lemma 3.3 we have for any $K>0$, as $u \rightarrow \infty$,

$$
\begin{aligned}
I_{2}(u, y) & \sim E\left[\sup _{t \geq 0}\left(\frac{l(0, t)}{1+t}\right)^{\alpha}\right] \int_{0}^{u K} v^{-1 / 2}(u+v)^{-\alpha / 2} \mathrm{~d} v \\
& =u^{(1 / 2)(1-\alpha)} E\left[\sup _{t \geq 0}\left(\frac{l(0, t)}{1+t}\right)^{\alpha}\right] \int_{0}^{K} x^{-1 / 2}(1+x)^{-\alpha / 2} \mathrm{~d} x .
\end{aligned}
$$

The desired result follows by letting $K \uparrow \infty$, taking (3.3) into account, and observing that

$$
\int_{0}^{\infty} x^{-1 / 2}(1+x)^{-\alpha / 2} \mathrm{~d} x=\beta\left(\frac{1}{2}, \frac{\alpha-1}{2}\right) .
$$

Proof of Theorem 3.1. By Proposition 3.1 it is sufficient to show the result for $\psi_{0}(u)$. For $u>0$ write

$$
\begin{aligned}
\frac{2 \psi_{0}(u)}{C_{\alpha}}= & \int_{\mathbb{R}} E\left[\sup _{t>0}\left(\frac{\int_{0}^{t} \varphi(B(s)+y) \mathrm{d} s}{u+t \mu}\right)^{\alpha}\right] \mathrm{d} y \\
= & \int_{-\infty}^{-1} E\left[\sup _{t>0}\left(\frac{\int_{0}^{t} \varphi(B(s)+y) \mathrm{d} s}{u+t \mu}\right)^{\alpha}\right] \mathrm{d} y \\
& +\int_{-1}^{1} E\left[\sup _{t>0}\left(\frac{\int_{0}^{t} \varphi(B(s)+y) \mathrm{d} s}{u+t \mu}\right)^{\alpha}\right] \mathrm{d} y \\
& +\int_{1}^{\infty} E\left[\sup _{t>0}\left(\frac{\int_{0}^{t} \varphi(B(s)+y) \mathrm{d} s}{u+t \mu}\right)^{\alpha}\right] \mathrm{d} y \\
= & : I_{1}(u)+I_{2}(u)+I_{3}(u) .
\end{aligned}
$$

Start by noting that, by Hölder's inequality we have

$$
\begin{aligned}
\limsup _{u \rightarrow \infty} u^{(1 / 2)(\alpha-1)} I_{2}(u) & =\limsup _{u \rightarrow \infty} u^{(1 / 2)(\alpha-1)} \int_{-1}^{1} E\left[\sup _{t>0}\left(\frac{\int_{\mathbb{R}} \varphi(x+y) l(x+t) \mathrm{d} x}{u+t \mu}\right)^{\alpha}\right] \mathrm{d} y \\
& \leq 2 \limsup _{u \rightarrow \infty} u^{(1 / 2)(\alpha-1)} E\left[\sup _{t>0}\left(\frac{\int_{-2}^{2} l(x, t) \mathrm{d} x}{u+t \mu}\right)^{\alpha}\right] \\
& \leq 2^{2 \alpha-1} \limsup _{u \rightarrow \infty} u^{(1 / 2)(\alpha-1)} E\left[\sup _{t>0} \frac{\int_{-2}^{2} l^{\alpha}(x, t) \mathrm{d} x}{(u+t \mu)^{\alpha}}\right]
\end{aligned}
$$

and, therefore, by (3.1) and the fact that the supremum of the local time $l(x, t)$, for all real $x$ 
and $t$ in a compact set, has moments of all orders finite, we have

$$
\begin{aligned}
\limsup _{u \rightarrow \infty} u^{(1 / 2)(\alpha-1)} I_{2}(u) & \leq 2^{2 \alpha-1} \limsup _{u \rightarrow \infty} u^{(1 / 2)(\alpha-1)} E\left[\sup _{t>0} \frac{t^{\alpha / 2} \int_{-2}^{2} l^{\alpha}(x / \sqrt{t}, 1) \mathrm{d} x}{(u+t \mu)^{\alpha}}\right] \\
& \leq \text { (constant) } \limsup _{u \rightarrow \infty} u^{(1 / 2)(\alpha-1)} \sup _{t>0}\left(\frac{\sqrt{t}}{u+t \mu}\right)^{\alpha} \\
& =\text { (constant) } \limsup _{u \rightarrow \infty} u^{(1 / 2)(\alpha-1)}\left(\frac{\sqrt{u / \mu}}{2 u}\right)^{\alpha} \\
& =0 .
\end{aligned}
$$

Let $\tau[y]:=\inf \{t \geq 0 ; B(t)=y\}$ be the first passage time to a level $y \in \mathbb{R}$, and observe that

$$
\begin{aligned}
I_{1}(u) & =\int_{-\infty}^{-1} E\left[\sup _{t>\tau[-1-y]}\left(\frac{\int_{0}^{t} \varphi(B(s)+y) \mathrm{d} s}{u+t \mu}\right)^{\alpha}\right] \mathrm{d} y \\
& =\int_{-\infty}^{-1} E\left[\sup _{t>0}\left(\frac{\int_{0}^{t+\tau[-1-y]} \varphi(B(s)+y) \mathrm{d} s}{u+(t+\tau[-1-y]) \mu}\right)^{\alpha}\right] \mathrm{d} y \\
& =\int_{-\infty}^{-1} E\left[\sup _{t>0}\left(\frac{\int_{\tau[-1-y]}^{t+\tau[-1-y]} \varphi(B(s)+y) \mathrm{d} s}{u+(t+\tau[-1-y]) \mu}\right)^{\alpha}\right] \mathrm{d} y .
\end{aligned}
$$

Also, recall that, for $v>0$ and $y \in \mathbb{R}$,

$$
\mathrm{P}(\tau[y] \in \mathrm{d} v)=\frac{|y|}{\sqrt{2 \pi v^{3}}} \mathrm{e}^{-y^{2} / 2 v} \mathrm{~d} v
$$

(cf. [11, p. 80]). Then it follows, from the strong Markov property for Brownian motion and Fubini's theorem, that

$$
\begin{aligned}
I_{1}(u) & =\int_{-\infty}^{-1} \int_{0}^{\infty} E\left[\sup _{t>0}\left(\frac{\int_{0}^{t} \varphi(B(s)-1) \mathrm{d} s}{u+(t+v) \mu}\right)^{\alpha}\right] \mathrm{P}(\tau[-1-y] \in \mathrm{d} v) \mathrm{d} y \\
& =\int_{0}^{\infty} E\left[\sup _{t>0}\left(\frac{\int_{0}^{t} \varphi(B(s)-1) \mathrm{d} s}{u+(t+v) \mu}\right)^{\alpha}\right] \int_{-\infty}^{-1} \frac{-1-y}{\sqrt{2 \pi v^{3}}} \mathrm{e}^{-(-1-y)^{2} / 2 v} \mathrm{~d} y \mathrm{~d} v \\
& =\frac{1}{\mu^{\alpha} \sqrt{2 \pi}} \int_{0}^{\infty} g\left(v+\frac{u}{\mu},-1\right) v^{-1 / 2} \mathrm{~d} v .
\end{aligned}
$$

Similarly,

Finally, note that

$$
I_{3}(u)=\frac{1}{\mu^{\alpha} \sqrt{2 \pi}} \int_{0}^{\infty} g\left(v+\frac{u}{\mu}, 1\right) v^{-1 / 2} \mathrm{~d} v .
$$

$$
I_{1}(u)=\frac{1}{\mu^{\alpha} \sqrt{2 \pi}} I\left(\frac{u}{\mu},-1\right) \text { and } I_{3}(u)=\frac{1}{\mu^{\alpha} \sqrt{2 \pi}} I\left(\frac{u}{\mu}, 1\right),
$$

and, hence, recalling (3.4) and using Lemma 3.4 we have

$$
\begin{aligned}
\frac{2 \psi_{0}(u)}{C_{\alpha}} & =\frac{1}{\mu^{\alpha} \sqrt{2 \pi}}\left[I\left(\frac{u}{\mu},-1\right)+I\left(\frac{u}{\mu}, 1\right)\right]+o\left(u^{(1 / 2)(1-\alpha)}\right) \\
& \sim \frac{2}{\sqrt{2 \pi} \mu^{(1 / 2)(\alpha+1)}} E\left[\sup _{t \geq 0}\left(\frac{l(0, t)}{1+t}\right)^{\alpha}\right] \beta\left(\frac{1}{2}, \frac{\alpha-1}{2}\right) u^{(1 / 2)(1-\alpha)}, \quad u \rightarrow \infty .
\end{aligned}
$$


Remark 3.1. All the results of this section prior to Theorem 3.1 are valid for general $H \in(0,1)$. This fact, together with the observation of parallels between the main results of this section and the previous section, lead us to believe that the result given in Theorem 3.1 should still hold with $\frac{1}{2}$ replaced by any $H \in(0,1)$. However, our proof requires the use of the strong Markov property which is only valid in the case where $H=\frac{1}{2}$.

\section{Acknowledgements}

This research was supported in part by NSF training grant 'Graduate and Postdoctoral Training in Probability and Its Applications', NSF grant DMS-0303493, and NSA grant MSPF05G-049.

\section{References}

[1] AdLer, R. J. (1990). An Introduction to Continuity, Extrema, and Related Topics for General Gaussian Processes (Inst. Math. Statist. Lecture Notes Monogr. Ser. 12). Institute of Mathematical Statistics, Hayward, CA.

[2] Alparslan, U. T. and Samorodnitsky, G. (2006). Asymptotic analysis of exceedance probability with stationary stable steps generated by dissipative flows. To appear in Scand. Actuarial J.

[3] Berman, S. M. (1973). Local nondeterminism and local times of Gaussian processes. Indiana Univ. Math. J. 23, 69-94.

[4] Braverman, M. (2004). Tail probabilities of subadditive functionals on stable processes with continuous and discrete time. Stoch. Process. Appl. 112, 157-183.

[5] Cohen, S. And Samorodnitsky, G. (2006). Random rewards, fractional Brownian local times and stable selfsimilar processes. Ann. Appl. Prob. 16, 1432-1461.

[6] Cramér, H.(1926). Review of F. Lundberg's "Försäkringsteknisk riskutjämning". Skand. Aktuarietidskr.

[7] Cramér, H. (1930). On the mathematical theory of risk. Skandia Jubilee, Stockholm.

[8] Embrechts, P. and Maejima, M. (2002). Selfsimilar Processes. Princeton University Press.

[9] Embrechts, P. and Veraverbeke, N. (1982). Estimates for the probability of ruin with special emphasis on the possibility of large claims. Insurance Math. Econom. 1, 55-72.

[10] Embrechts, P., KlüPpelberg, C. And Mikosch, T. (1997). Modelling Extremal Events (Appl. Math. (New York) 33). Springer, Berlin.

[11] Karatzas, I. and Shreve, S. E. (1991). Brownian Motion and Stochastic Calculus (Graduate Texts Math. 113), 2nd edn. Springer, New York.

[12] Krengel, U. (1985). Ergodic Theorems (De Gruyter Studies Math. 6). De Gruyter, Berlin.

[13] LundBerg, F. (1903). I approximerad framställning av sannolikhetsfunktionen. ii återförsäkring av kollektivrisker. Doctoral Thesis, Akad. Afhandling. Almqvist och Wiksell, Uppsala.

[14] Lundberg, F. (1909). Über die theorie der rückversicherung. Trans. VI Int. Congr. Actuaries 1, 877-955.

[15] Lundberg, F. (1926). Försäkringsteknisk Riskutjämning. F. Englunds Boktryckeri AB, Stockholm.

[16] Mikosch, T. And Samorodnitsky, G. (2000). Ruin probability with claims modeled by a stationary ergodic stable process. Ann. Prob. 28, 1814-1851.

[17] ReSnick, S. (1992). Adventures in Stochastic Processes. Birkhäuser, Boston, MA.

[18] Resnick, S. I. (1999). A Probability Path. Birkhäuser, Boston, MA.

[19] Resnick, S., Samorodnitsky, G. And Xue, F. (1999). How misleading can sample ACFs of stable MAs be? (Very!). Ann. Appl. Prob. 9, 797-817.

[20] Rosiński, J. (1995). On the structure of stationary stable processes. Ann. Prob. 23, 1163-1187.

[21] Rosiński, J. And SAmorodnitsky, G. (1996). Classes of mixing stable processes. Bernoulli 2, 365-377.

[22] Samorodnitsky, G. (2004). Maxima of continuous-time stationary stable processes. Adv. Appl. Prob. 36, 805-823.

[23] Samorodnitsky, G. and Taqqu, M. S. (1994). Stable Non-Gaussian Random Processes. Chapman \& Hall, New York.

[24] Teugels, J. L. (1970). Regular variation of Markov renewal functions. J. London Math. Soc. (2) 2, 179-190. 